\title{
Model-based control of intelligent traffic networks*
}

\author{
B. De Schutter, H. Hellendoorn, A. Hegyi, M. van den Berg, and
} S.K. Zegeye

If you want to cite this report, please use the following reference instead: B. De Schutter, H. Hellendoorn, A. Hegyi, M. van den Berg, and S.K. Zegeye, "Model-based control of intelligent traffic networks," Chapter 11 in Intelligent Infrastructures (R.R. Negenborn, Z. Lukszo, and H. Hellendoorn, eds.), vol. 42 of Intelligent Systems, Control and Automation: Science and Engineering, Dordrecht, The Netherlands: Springer, ISBN 978-90-481-3598-1, pp. 277-310, 2010.

Delft Center for Systems and Control Delft University of Technology

Mekelweg 2, 2628 CD Delft

The Netherlands

phone: +31-15-278.24.73 (secretary)

URL: https: //www.dcsc.tudelft.nl

${ }^{*}$ This report can also be downloaded via https://pub.deschutter.info/abs/09_020.html 


\title{
Model-based control of intelligent traffic networks
}

B. De Schutter, H. Hellendoorn, A. Hegyi, M. van den Berg, and S.K. Zegeye

\begin{abstract}
Road traffic networks are increasingly being equipped and enhanced with various sensing, communication, and control units, resulting in an increased intelligence in the network and offering additional handles for control. In this chapter we discuss some advanced model-based control methods for intelligent traffic networks. In particular, we consider model predictive control (MPC) of integrated freeway and urban traffic networks. We present the basic principles of MPC for traffic control including prediction models, control objectives, and constraints. The proposed MPC control approach is modular, allowing the easy substitution of prediction models and the addition of extra control measures or the extension of the network. Moreover, it can be used to obtain a balanced trade-off between various objectives such as throughput, emissions, noise, fuel consumption, etc. Moreover, MPC also allows the integration and network-wide coordination of various traffic control measures such as traffic signals, speed limits, ramp metering, lane closures, etc. We illustrate the MPC approach for traffic control with two case studies. The first case study involves control of a freeway stretch with a balanced trade-off between total time spent, fuel consumption, and emissions as control objective. The second case study has a more complex layout and involves control of a mixed urban-freeway network with total time spent as control objective.
\end{abstract}

B. De Schutter

Delft Center for Systems and Control \& Marine and Transport Technology, Delft University of Technology, e-mail: b@deschutter.info

H. Hellendoorn, M. van den Berg, S.K. Zegeye

Delft Center for Systems and Control, Delft University of Technology, e-mail: \{j.hellendoorn,monique.vandenberg,s.k.zegeye\}@tudelft.nl

A. Hegyi

Department of Transport \& Planning, Faculty of Civil Engineering and Geosciences, Delft University of Technology, e-mail: a.hegyi@tudelft.nl 


\section{Introduction}

\subsection{Positioning and relation of intelligent traffic networks with other networks}

Each infrastructure has its own characteristics. Electricity networks are governed by the laws of Kirchhoff, which state that voltage and current distribute equally over the network. Water networks are governed by the law of gravity, which states that water is always flowing downwards, unless one makes use of pumping stations. Gas networks are determined by pressure, telecommunication networks by the behavior of the users. In all these cases the subjects that are transported are passive: electrons, water, gas molecules, and bits and bytes do not have an own will. Road networks form a special class, because the subjects, the drivers, are self-willed and do not always obey the proposed traffic measures. Nevertheless, the road network is too important to let it be uncontrolled. Economics and society depend heavily on efficient roads. For instance, in the European Union $44 \%$ of all goods are moved by trucks over roads and $85 \%$ of all persons are transported by cars, buses, or coaches on the roads.

There are several aspects of the road network that have to be mentioned. Firstly, the possible measures that can be taken and the limitations of these measures to control individual drivers. Secondly, the role of governments and other (nongovernment) parties in traffic control, in particular with regards to traffic jams and environmental issues. Thirdly, the road network as a critical infrastructure that is vital for the economy. And fourthly, the expectation of modern societies for flexible road networks without frequent construction works.

Until half a century ago streets and roads were passive infrastructures. The main function of the infrastructure was to facilitate comfortable and quick driving. But the number of vehicles has increased significantly over the last decades, which has led to traffic jams and dangerous situations on crossings. So traffic signals were introduced, first with a fixed-time scheme, and later on with computer programs connected to induction loops in the streets. Moreover, on highways variable speed limits and ramp metering were introduced, nowadays combined with Dynamic Route Information Panels (DRIPs). The behavior of drivers towards the road signs has changed: they now expect that traffic control measures can oversee the total traffic situation and, at the same time, have a good understanding of the needs of individual drivers, such that the drivers are not waiting seemingly meaningless for a traffic signal or a ramp metering installation, or have to drive slower than meaningful on a highway. Due to improved car mechanics, safer cars, and better roads, drivers have adapted their driving behavior, e.g., by keeping less distance to each other. In emergency cases this can lead to dangerous traffic situations and severe accidents.

Governments play a large role in defining targets for traffic management. Traffic safety has become a main political issue leading to new road constructions, separate lanes, and more roundabouts. Governments have clear targets to reduce the number of road casualties. They are also under severe pressure to reduce traffic jams, which 
leads, e.g., to the use of shoulder lanes in combination with camera surveillance during rush hours. Furthermore, legislation on environmental protection forces governments to take actions against pollution of $\mathrm{CO}_{2}, \mathrm{NO}_{\mathrm{x}}, \mathrm{HC}$, and particulate matters. Interest groups of truck drivers, automobile clubs, ecology movements, and neighboring residents and companies also play an important role in the public discussion about road constructions and traffic management.

Roads as well as electricity and drinking water networks have become critical infrastructures. They are of vital importance for the well-functioning of modern society. Traffic jams have large economical impact that may sum up to several percentages of the Gross Domestic Product (GDP), especially in (parts of) countries with a dense population where an accident on one of the main junctions can lead to a total collapse of the road network. In The Netherlands the length of traffic jams increased with $8.1 \%$ in 2007 , the number of traffic jams between 3.30 p.m. and 8.00 p.m. increased with $6.5 \%$ in the same period, and between $10.00 \mathrm{a} . \mathrm{m}$. and 3.30 p.m. the length of traffic jams increased by even $22.8 \%$. Worldwide, traffic incidents cost approximately 1-2\% of the GDP, i.e., approximately 65 billion dollar. Road congestion amounts to an average $1 \%$ of GDP in the European Union, with Great Britain and France at $1.5 \%$. So reducing congestion contributes to a healthier economy.

Society demands more functionality, capacity, and quality of the road network. Construction works are necessary, but should influence the traffic flow as little as possible. Road networks should be flexible on the long term: it should be possible to quickly implement new political or societal desires. Extensions of the network or new driving concepts like adaptive cruise control or platooning should not lead to construction works that last many years.

There are several (partial) solutions to address all the issues discussed above, and one of them is the use of intelligent dynamic traffic management, which is the topic of this chapter.

\subsection{The need for intelligent dynamic traffic management}

As already indicated above, the need for mobility is increasing due to the growing number of road users as well as the increasing number of movements per user [36]. This leads to an increase in the frequency, length, and duration of traffic jams. These traffic jams cause large delays, resulting in higher travel costs and they also have a negative impact on the environment due to, e.g., noise and pollution.

To tackle these congestion problems there exist different solution approaches: constructing new roads, levying tolls, promoting public transport, or making more efficient use of the existing infrastructure. In this chapter we consider the last approach, implemented using dynamic traffic management and traffic control measures, such as on-ramp metering, dynamic speed limits, traffic signals, dynamic routing, provision of congestion information, etc., since this solution is effective on the short term, and inexpensive compared to constructing new roads. In addition, 
it is flexible enough to deal with the new infrastructures that will be constructed in the long term.

Current traffic control approaches usually focus on either urban traffic or freeway traffic. In urban areas traffic signals are the most frequently used control measures. Traditionally, they are controlled locally using fixed-time settings, or they are vehicle-actuated, meaning that they react on the prevailing traffic situation. Nowadays sophisticated, dynamic systems that aim at coordinating different available control measures in order to improve the total performance, are also making progress: systems such as SCOOT [46], SCATS [58], Toptrac [3], TUC [13], Mitrop [17], Motion [8], and UTOPIA/SPOT [43] use a coordinated control method to improve the urban traffic circulation, e.g., by constructing green waves. Control on freeways is done using different traffic control measures. Ramp metering is applied on on-ramps, using methods like ALINEA [40]. Overviews of ramp metering methods and results are given in $[41,50]$. The use of variable speed limits on freeways is described in [2, 21, 32, 49], and the use of route guidance in [11, 13, 26]. Several authors have described methods for coordinated control of freeways using different traffic control measures [6, 19, 28, 29].

Usually these traffic control measures operate based on local data (occupancy, intensity, or speed measurements). However, considering the effect of the measures on the network level has many advantages compared to local control. E.g., solving a local traffic jam only may have as a consequence that the vehicles run faster into another (downstream) traffic jam, whereas still the same amount of vehicles have to pass the bottleneck (with a given capacity), and so the average travel time at the network level will still be the same. Another reason for considering the effects of control at the network level, is that in a dense network a local control measure can have effects on more distant parts of the network: an improved flow may cause congestion somewhere else in the network or a reduced flow may prevent congestion somewhere else in the network. Another source of degraded network performance is that congestion may block traffic flows on routes that do not pass the bottleneck (or incident location), such as a freeway with a congested off-ramp where the vehicles that want to leave the freeway block the mainstream traffic. Similar arguments also hold for urban and mixed urban-freeway traffic networks.

The traffic flows on freeways are often influenced by traffic flows on urban roads, and vice versa. Freeway control measures like ramp metering or speed limits allow a better flow and a larger throughput, but could lead to longer queues on on-ramps. These queues may spill back and block urban roads. On the other hand, urban traffic management policies often try to get vehicles on the freeway network as soon as possible, displacing the congestion toward neighboring freeways. The problems between the two road types are often increased by the fact that in several countries urban roads and freeways are managed by different traffic authorities, each with their own policies and objectives.

Hence, there is a clear need for coordinated and network-wide traffic management and control. Therefore, we present a coordinated control approach for mixed urban-freeway networks that provides an appropriate trade-off between the perfor- 
mance of the urban and freeway traffic operations, and that results in a significant improvement of the performance of the overall network.

As control method we use a model predictive control (MPC) approach [9, 34], adapted for traffic control. MPC is an online model-based predictive control approach in which a prediction model and (online) optimization are used to determine the control actions that optimize a given performance criterion over a given time horizon subject to given constraints. Using a receding horizon approach, only the first step of the computed control signal is applied, and next the optimization is started again with the prediction horizon shifted one time step further. MPC has already been applied to coordinated control of freeway networks in $[6,19,29]$. In this chapter, which collects and extends several of our previous results reported in [19$21,52-54,59,60]$, we demonstrate how MPC can be used to obtain coordinated and integrated control of traffic networks containing both freeways and urban roads as well as a wide variety of traffic control measures, such that a balanced trade-off is obtained between various performance criteria and such that (hard) constraints are taken into account. Other publications that deal with MPC or MPC-like approaches for traffic control are [12, 16, 29, 43].

\subsection{Overview of the chapter}

This chapter is organized as follows. We first present an integrated traffic flow model for networks that contain both urban roads and freeways, as well as an emission and fuel consumption model in Section 2. Next, we describe MPC-based control for intelligent traffic networks in Section 3. The proposed approach is then illustrated in Section 4 via two simple case studies: one involving the balanced optimization of total time spent, total fuel consumption, and total emissions, and one involving a mixed urban-freeway network with total time spent as cost criterion. Section 5 concludes the chapter.

\section{Traffic models}

Traffic flow models can be distinguished according to the level of detail they use to describe the traffic. An overview of existing traffic models is given in [25].

In this chapter we use macroscopic traffic models. Macroscopic models describe traffic flows using aggregated variables such as flows and densities. They are suited very well for online control since these models give a balanced trade-off between accurate predictions and computational efforts. Indeed, the computation time for a macroscopic model does not depend on the number of vehicles in the network, which makes the model well-suited for online control, where the prediction should be performed online in an optimization setting, requiring that the model should run several times faster than real-time. Examples of macroscopic models are the LWR 
model [33, 45], the models of Helbing [22] and Hoogendoorn [24], and METANET [35].

We use an extended version of the METANET traffic flow model [35] to describe the freeway traffic, and a modified and extended model based on a queue length model developed by Kashani and Saridis [27] for the urban traffic. We also discuss how the freeway and the urban model have to be coupled, and we explain how the resulting traffic flow model can be linked with a model that describes emissions and fuel consumption. This leads to a macroscopic traffic model for mixed networks with urban roads and freeways, especially suited for an MPC-based traffic control approach.

Note that we will explicitly make a difference between the simulation time step $T_{\mathrm{f}}$ for the freeway part of the network, the simulation time step $T_{\mathrm{u}}$ for the urban part of the network, and the controller sample time $T_{\mathrm{c}}$. We will also use three different counters: $k_{\mathrm{f}}$ for the freeway part, $k_{\mathrm{u}}$ for the urban part, and $k_{\mathrm{c}}$ for the controller. For simplicity, we assume that $T_{\mathrm{u}}$ is an integer divisor of $T_{\mathrm{f}}$, and that $T_{\mathrm{f}}$ is an integer divisor of $T_{\mathrm{c}}$ :

$$
T_{\mathrm{f}}=M_{\mathrm{fu}} T_{\mathrm{u}}, T_{\mathrm{c}}=M_{\mathrm{cf}} T_{\mathrm{f}}=M_{\mathrm{cf}} M_{\mathrm{fu}} T_{\mathrm{u}},
$$

with $M_{\mathrm{fu}}$ and $M_{\mathrm{cf}}$ integers. The value for $T_{\mathrm{f}}$ must be selected in such a way that no vehicle can cross a freeway segment in one time step, which results in a typical value of $10 \mathrm{~s}$ for freeway segments of length $0.5 \mathrm{~km}$. The value of $T_{\mathrm{u}}$ is in general selected small enough to obtain an accurate description of the traffic, typically between 1 and $5 \mathrm{~s}$, depending on the length of the roads. The control time step $T_{\mathrm{c}}$ should be large enough to allow the traffic controller to determine the new control signal, which depends on the required computation time, and short enough to deal with changing traffic conditions. Typical values for $T_{\mathrm{c}}$ are 1-5 min.

\subsection{Freeway traffic flow model}

For the prediction of the traffic flows on the freeway part of the network we use the destination-independent METANET model from [29, 30, 39]. We will briefly present the basic METANET model here. For a full description we refer the interested reader to [29, 30, 39].

The METANET model represents a network as a directed graph with the links (indicated by the index $m$ ) corresponding to freeway stretches. Each freeway link has uniform characteristics, i.e., no on-ramps or off-ramps and no major changes in geometry. Where major changes occur in the characteristics of the link or in the road geometry (e.g., at an on-ramp or an off-ramp), a node is placed. Each link $m$ is divided into $N_{m}$ segments (indicated by the index $i$ ) of length $L_{m}$ (see Figure 1). Each segment $i$ of link $m$ is characterized by the traffic density $\rho_{m, i}\left(k_{\mathrm{f}}\right)(\mathrm{veh} / \mathrm{km} / \mathrm{lane})$, the mean speed $v_{m, i}\left(k_{\mathrm{f}}\right)(\mathrm{km} / \mathrm{h})$, and the traffic volume or outflow $q_{m, i}\left(k_{\mathrm{f}}\right)(\mathrm{veh} / \mathrm{h})$, where 


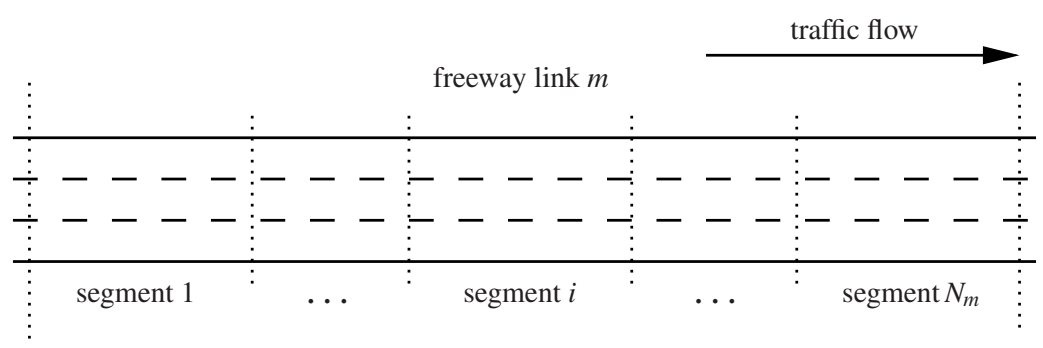

Fig. 1 In the METANET model a freeway link is divided into segments.

$k_{\mathrm{f}}$ indicates the time instant $t=k_{\mathrm{f}} T_{\mathrm{f}}$, and $T_{\mathrm{f}}$ is the time step used for the simulation of the freeway traffic flow (typically $T_{\mathrm{f}}=10 \mathrm{~s}$ ).

The following equations describe the evolution of the network over time. The outflow of each segment is equal to the density multiplied by the mean speed and the number of lanes on that segment (denoted by $\lambda_{m}$ ):

$$
q_{m, i}\left(k_{\mathrm{f}}\right)=\rho_{m, i}\left(k_{\mathrm{f}}\right) v_{m, i}\left(k_{\mathrm{f}}\right) \lambda_{m} .
$$

The density of a segment equals the previous density plus the inflow from the upstream segment, minus the outflow of the segment itself (conservation of vehicles):

$$
\rho_{m, i}\left(k_{\mathrm{f}}+1\right)=\rho_{m, i}\left(k_{\mathrm{f}}\right)+\frac{T_{\mathrm{f}}}{L_{m} \lambda_{m}}\left(q_{m, i-1}\left(k_{\mathrm{f}}\right)-q_{m, i}\left(k_{\mathrm{f}}\right)\right) .
$$

While (2) and (3) are based on physical principles and are exact, the equations that describe the speed dynamics and the relation between density and the desired speed are heuristic. The mean speed at step $k_{\mathrm{f}}+1$ equals the mean speed at step $k_{\mathrm{f}}$ plus a relaxation term that expresses that the drivers try to achieve a desired speed $V$, a convection term that expresses the speed increase (or decrease) caused by the inflow of vehicles, and an anticipation term that expresses the speed decrease (increase) as drivers experience a density increase (decrease) downstream:

$$
\begin{aligned}
& v_{m, i}\left(k_{\mathrm{f}}+1\right)=v_{m, i}\left(k_{\mathrm{f}}\right)+\frac{T_{\mathrm{f}}}{\tau}\left(V\left(\rho_{m, i}\left(k_{\mathrm{f}}\right)\right)-v_{m, i}\left(k_{\mathrm{f}}\right)\right)+ \\
& \frac{T_{\mathrm{f}}}{L_{m}} v_{m, i}\left(k_{\mathrm{f}}\right)\left(v_{m, i-1}\left(k_{\mathrm{f}}\right)-v_{m, i}\left(k_{\mathrm{f}}\right)\right)-\frac{\vartheta T_{\mathrm{f}}}{\tau L_{m}} \frac{\rho_{m, i+1}\left(k_{\mathrm{f}}\right)-\rho_{m, i}\left(k_{\mathrm{f}}\right)}{\rho_{m, i}\left(k_{\mathrm{f}}\right)+\kappa},
\end{aligned}
$$

where $\tau, \vartheta$, and $\kappa$ are model parameters. The expression for the desired speed $V$ is given by

$$
V\left(\rho_{m, i}\left(k_{\mathrm{f}}\right)\right)=v_{\text {free }, m} \cdot \exp \left[-\frac{1}{a_{m}}\left(\frac{\rho_{m, i}\left(k_{\mathrm{f}}\right)}{\rho_{\text {crit }, m}}\right)^{a_{m}}\right],
$$


with $a_{m}$ a model parameter, and where the free-flow speed $v_{\text {free, } m}$ is the average speed that drivers assume if traffic is freely flowing, and the critical density $\rho_{\text {crit }, m}$ is the density at which the traffic flow is maximal.

When a speed limit is active in the segment, (5) becomes

$$
V\left(\rho_{m, i}\left(k_{\mathrm{f}}\right)\right)=\min \left(v_{\mathrm{free}, m} \cdot \exp \left[-\frac{1}{a_{m}}\left(\frac{\rho_{m, i}(k)}{\rho_{\mathrm{crit}, m}}\right)^{a_{m}}\right],(1+\alpha) v_{\mathrm{control}, m, i}\left(k_{\mathrm{f}}\right)\right),
$$

where $v_{\text {control }, m, i}\left(k_{\mathrm{f}}\right)$ is the speed limit imposed on segment $i$ of link $m$ at time step $k_{\mathrm{f}}$, and $1+\alpha$ is the non-compliance factor that expresses that drivers usually do not fully comply with the displayed speed limit and that their target speed is usually higher than what is displayed ${ }^{1}$.

Origins are modeled with a simple queue model. The length of the queue equals the previous queue length plus the demand $d_{o}\left(k_{\mathrm{f}}\right)$, minus the outflow $q_{o}\left(k_{\mathrm{f}}\right)$ :

$$
w_{o}\left(k_{\mathrm{f}}+1\right)=w_{o}\left(k_{\mathrm{f}}\right)+T_{\mathrm{f}}\left(d_{o}\left(k_{\mathrm{f}}\right)-q_{o}\left(k_{\mathrm{f}}\right)\right) .
$$

The outflow of the origin depends on the traffic conditions on the mainstream and, for the metered on-ramp, on the ramp metering rate $^{2} r_{o}\left(k_{\mathrm{f}}\right)$, where $r_{o}\left(k_{\mathrm{f}}\right) \in$ $[0,1]$. More specifically, $q_{o}\left(k_{\mathrm{f}}\right)$ is the minimum of three quantities: the available traffic in time period $k_{\mathrm{f}}$ (queue plus demand), the maximal flow that could enter the freeway because of the mainstream conditions, and the maximal flow allowed by the metering rate:

$$
q_{o}\left(k_{\mathrm{f}}\right)=\min \left[d_{o}\left(k_{\mathrm{f}}\right)+\frac{w_{o}\left(k_{\mathrm{f}}\right)}{T_{\mathrm{f}}}, Q_{o} \cdot r_{o}\left(k_{\mathrm{f}}\right), Q_{o}\left(\frac{\rho_{\max , m}-\rho_{m, 1}\left(k_{\mathrm{f}}\right)}{\rho_{\max , m}-\rho_{\mathrm{crit}, m}}\right)\right],
$$

where $Q_{o}$ is the on-ramp capacity (veh/h) under free-flow conditions and $\rho_{\max , m}$ (veh/km/lane) is the maximum density of link $m$, and $m$ is the index of the link to which the on-ramp is connected.

The above equations can be extended with terms that account for the speed drop caused by merging phenomena if there is an on-ramp, or for the speed reduction due to weaving phenomena when there is a lane drop, see [29].

The coupling equations to connect links are as follows. Every time there is a major change in the link parameters, like a junction or a bifurcation, a node is placed between the links. This node provides the incoming links with a virtual downstream density (required for the speed update equation (4)), and the leaving links with an inflow and a virtual upstream speed (required for the density update equation (3) and the speed update equation (4)). The flow that enters node $n$ is distributed among the leaving links according to

${ }^{1}$ Data from the Dutch freeways show that when the speed limits are not enforced the average speed is approximately $10 \%$ higher than what is displayed $(\alpha=0.1)$, and when they are enforced the average speed is approximately $10 \%$ lower than what is displayed $(\alpha=-0.1)$.

${ }^{2}$ For an unmetered on-ramp we can also use (8) by setting $r_{o}\left(k_{\mathrm{f}}\right) \equiv 1$. 


$$
\begin{aligned}
Q_{n}\left(k_{\mathrm{f}}\right) & =\sum_{\mu \in I_{n}} q_{\mu, N_{\mu}}\left(k_{\mathrm{f}}\right) \\
q_{m, 0}\left(k_{\mathrm{f}}\right) & =\beta_{m, n}\left(k_{\mathrm{f}}\right) \cdot Q_{n}\left(k_{\mathrm{f}}\right),
\end{aligned}
$$

where $Q_{n}\left(k_{\mathrm{f}}\right)$ is the total flow that enters the node at step $k_{\mathrm{f}}, I_{n}$ is the set of links that enter node $n, \beta_{m, n}\left(k_{\mathrm{f}}\right)$ expresses the turning rates (i.e., the fraction of the total flow through node $n$ that leaves via link $m$ ), and $q_{m, 0}\left(k_{\mathrm{f}}\right)$ is the flow that leaves node $n$ via link $m$.

When node $n$ has more than one leaving link, the virtual downstream density $\rho_{m, N_{m}+1}\left(k_{\mathrm{f}}\right)$ of entering link $m$ is given by

$$
\rho_{m, N_{m}+1}\left(k_{\mathrm{f}}\right)=\frac{\sum_{\mu \in O_{n}} \rho_{\mu, 1}^{2}\left(k_{\mathrm{f}}\right)}{\sum_{\mu \in O_{n}} \rho_{\mu, 1}\left(k_{\mathrm{f}}\right)},
$$

where $O_{n}$ is the set of links leaving node $n$.

When node $n$ has more than one entering link, the virtual upstream speed $v_{m, 0}\left(k_{\mathrm{f}}\right)$ of leaving link $m$ is given by

$$
v_{m, 0}\left(k_{\mathrm{f}}\right)=\frac{\sum_{\mu \in I_{n}} v_{\mu, N_{\mu}}\left(k_{\mathrm{f}}\right) \cdot q_{\mu, N_{\mu}}\left(k_{\mathrm{f}}\right)}{\sum_{\mu \in I_{n}} q_{\mu, N_{\mu}}\left(k_{\mathrm{f}}\right)} .
$$

Extensions to the basic METANET model are presented in [19-21].

\subsection{Urban traffic flow model}

Now we present a macroscopic model that describes the evolution of the traffic flows in the urban part of the network. This model is based on the Kashani model [27], but with the following extensions:

1. Horizontal, turning-direction-dependent queues;

2. Blocking effects, represented by maximal queue lengths and a flow constraint on flows that want to enter the blocked link, so no vehicle will be able to cross a blocked intersection;

3. A shorter time step $^{3}$, to get a more accurate description of the traffic flows.

The main variables used in the urban model are shown in Figures 2(a) and 2(b). The most important variables are the queue length $x$ expressed in number of vehicles, the number of arriving vehicles $m_{\text {arr }}$, and the number of departing vehicles $m_{\text {dep }}$. Using these variables, the model is formulated as follows.

The number of vehicles that intend to leave the link $l_{o_{i}, s}$, connecting origin $o_{i}$ and intersection $s$, toward destination $d_{j}$ at time $t=k_{\mathrm{u}} T_{\mathrm{u}}$ is given by:

\footnotetext{
3 The original Kashani model of [27] uses the cycle time as time step, which restricts the model to effects that take longer than the cycle time. For MPC-based traffic control the other effects can also be relevant, and one might also want to control the cycle times.
} 


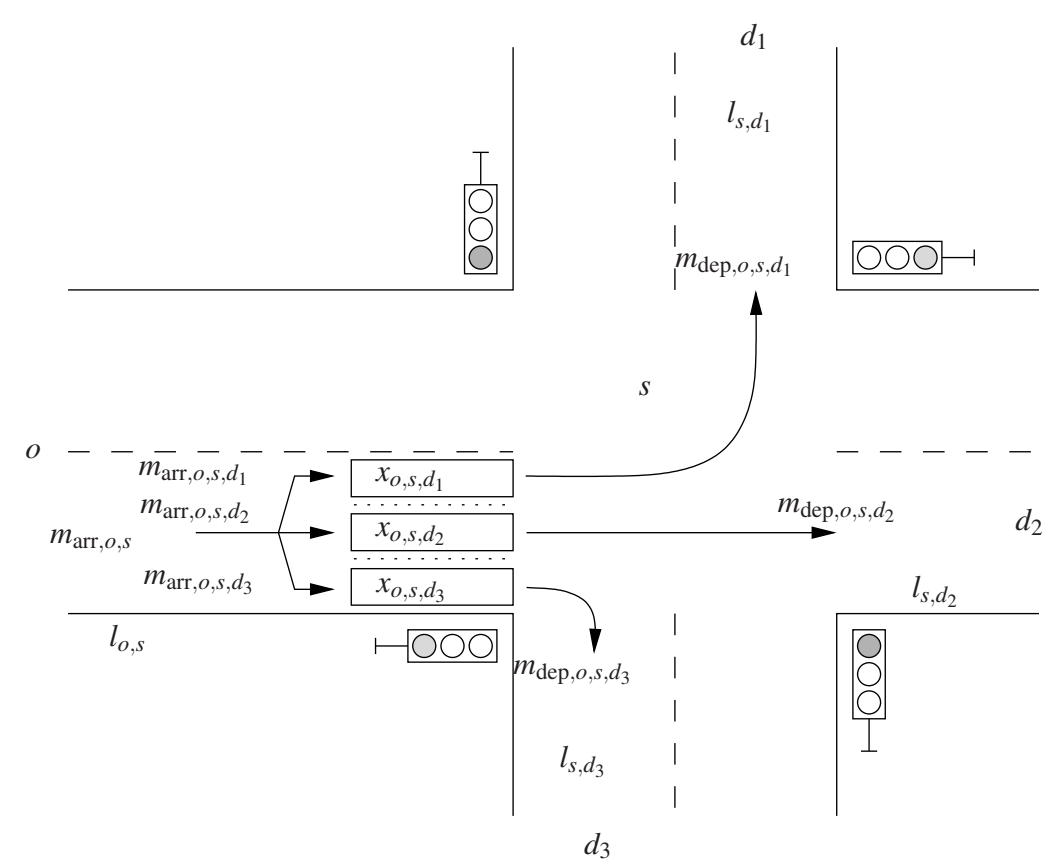

(a) Variables for an urban intersection.

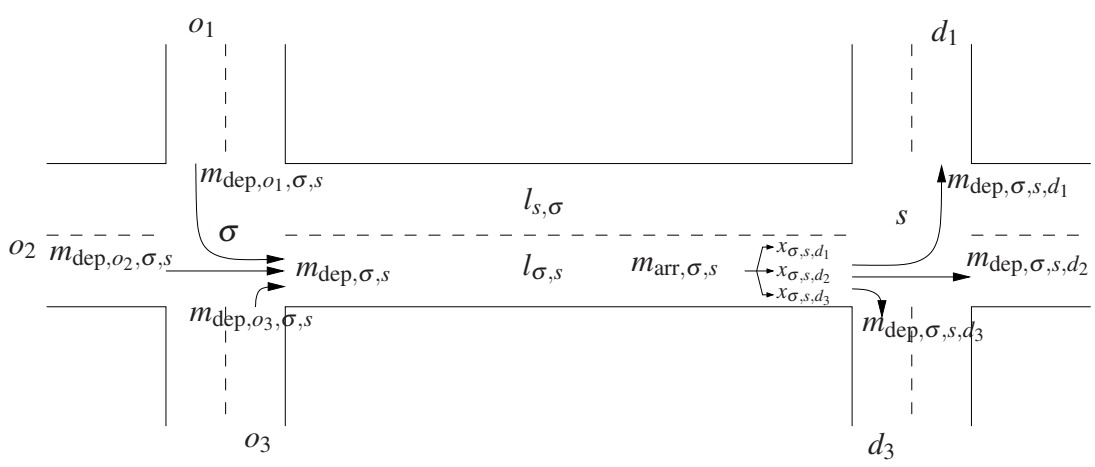

(b) Variables for an urban link.

Fig. 2 Overview of the urban network variables.

$$
\begin{aligned}
& m_{\mathrm{dep}, \text { int }, o_{i}, s, d_{j}}\left(k_{\mathrm{u}}\right)= \\
& \begin{cases}0 & \text { if } g_{o_{i}, s, d_{j}}\left(k_{\mathrm{u}}\right)=0, \\
\min \left(x_{o_{i}, s, d_{j}}\left(k_{\mathrm{u}}\right)+m_{\mathrm{arr}, o_{i}, s, d_{j}}\left(k_{\mathrm{u}}\right),\right. & \\
\left.S_{s, d_{j}}\left(k_{\mathrm{u}}\right), T_{\mathrm{u}} Q_{\mathrm{cap}, o_{i}, s, d_{j}}\right) & \text { if } g_{o_{i}, s, d_{j}}\left(k_{\mathrm{u}}\right)=1,\end{cases}
\end{aligned}
$$

where $T_{\mathrm{u}}$ is the urban step with $k_{\mathrm{u}}$ as counter, $x_{o_{i}, s, d_{j}}\left(k_{\mathrm{u}}\right)$ is the queue length consisting of vehicles coming from origin $o_{i}$ and going to destination $d_{j}$ at intersection $s$, 
$m_{\mathrm{arr}, o_{i}, s, d_{j}}\left(k_{\mathrm{u}}\right)$ is the number of vehicles arriving at the end of this queue, $S_{s, d_{j}}\left(k_{\mathrm{u}}\right)$ is the free space in the downstream link expressed in number of cars, $Q_{\text {cap }, o_{i}, s, d_{j}}$ is the saturation flow ${ }^{4}$, and $g_{o_{i}, s, d_{j}}\left(k_{\mathrm{u}}\right)$ is a binary signal that is 1 when the specified traffic direction has green, and zero otherwise. This means that $g_{o_{i}, s, d_{j}}=0$ corresponds to a red traffic signal, and $g_{o_{i}, s, d_{j}}=1$ to a green one. ${ }^{5}$

The free space $S_{\sigma, s}$ in a link $l_{\sigma, s}$ expresses the maximum number of vehicles that can enter the link. It can never be larger than the length $L_{\sigma, s}$ of the link expressed in number vehicles, and is computed as follows:

$$
S_{\sigma, s}\left(k_{\mathrm{u}}+1\right)=S_{\sigma, s}\left(k_{\mathrm{u}}\right)-m_{\mathrm{dep}, \sigma, s}\left(k_{\mathrm{u}}\right)+\sum_{d_{j} \in D_{s}} m_{\mathrm{dep}, \sigma, s, d_{j}}\left(k_{\mathrm{u}}\right),
$$

where $m_{\mathrm{dep}, \sigma, s}\left(k_{\mathrm{u}}\right)$ is the number of vehicles departing from intersection $\sigma$ towards link $l_{\sigma, s}$, and $D_{s}$ is the set of destinations connected to intersection $s$.

The number of vehicles departing from intersection $s$ towards link $l_{s, d_{j}}$ can be computed as

$$
m_{\mathrm{dep}, s, d_{j}}\left(k_{\mathrm{u}}\right)=\sum_{o_{i} \in O_{s}} m_{\mathrm{dep}, o_{i}, s, d_{j}}\left(k_{\mathrm{u}}\right)
$$

These vehicles drive from the beginning of the link $l_{s, d_{j}}$ toward the tail of the queue waiting on the link. This gives a time delay $\delta_{s, d_{j}}\left(k_{\mathrm{u}}\right)$ which is approximated as:

$$
\delta_{s, d_{j}}\left(k_{\mathrm{u}}\right)=\operatorname{ceil}\left(\frac{S_{s, d_{j}}\left(k_{\mathrm{u}}\right) L_{\mathrm{av}, \mathrm{veh}}}{v_{\mathrm{av}, s, d_{j}}}\right),
$$

where $L_{\mathrm{av}, \text { veh }}$ is the average length of a vehicle, and $v_{\mathrm{av}, s, d_{j}}$ the average speed on link $l_{s, d_{j}}$.

The time instant at which the vehicle enters the link and the vehicle's delay on the link result in the time instant at which the vehicle will arrive at the end of the queue. It can happen that vehicles that have entered the link at different instants reach the end of the queue during the same time step. To take this into account the variable $m_{\mathrm{arr}, s, d_{j}}\left(k_{\mathrm{u}}\right)$ that describes the vehicles arriving at the end of the queue is updated accumulatively every time step. This results in:

$$
m_{\mathrm{arr}, s, d_{j}}\left(k_{\mathrm{u}}+\delta_{s, d_{j}}\left(k_{\mathrm{u}}\right)\right)_{\text {new }}=m_{\mathrm{arr}, s, d_{j}}\left(k_{\mathrm{u}}+\delta_{s, d_{j}}\left(k_{\mathrm{u}}\right)\right)_{\mathrm{old}}+m_{\mathrm{dep}, s, d_{j}}\left(k_{\mathrm{u}}\right),
$$

where $m_{\mathrm{arr}, s, d_{j}}\left(k_{\mathrm{u}}+\delta_{s, d_{j}}\left(k_{\mathrm{u}}\right)\right)$ is the number of vehicles arriving at the end of the queue at time $k_{\mathrm{u}}+\delta_{s, d_{j}}\left(k_{\mathrm{u}}\right)$, and $m_{\mathrm{dep}, s, d_{j}}\left(k_{\mathrm{u}}\right)$ is the number of vehicles entering link $l_{s, d_{j}}$.

The traffic flow reaching the tail of the queue in link $l_{s, d_{j}}$ divides itself over the subqueues according to the turning rates $\beta_{o_{i}, s, d_{j}}\left(k_{\mathrm{u}}\right)$ :

\footnotetext{
4 The saturation flow is the maximum flow that can cross the intersection under free-flow conditions.

5 The computed green time is the effective green time. The exact signal timing including the amber time can easily be derived from this effective green time.
} 


$$
m_{\mathrm{arr}, o_{i}, s, d_{j}}\left(k_{\mathrm{u}}\right)=\beta_{o_{i}, s, d_{j}}\left(k_{\mathrm{u}}\right) m_{\mathrm{arr}, o_{i}, s}\left(k_{\mathrm{u}}\right) .
$$

The subqueues are then updated as follows:

$$
x_{o_{i}, s, d_{j}}\left(k_{\mathrm{u}}+1\right)=x_{o_{i}, s, d_{j}}\left(k_{\mathrm{u}}\right)+m_{\mathrm{arr}, o_{i}, s, d_{j}}\left(k_{\mathrm{u}}\right)-m_{\mathrm{dep}, o_{i}, s, d_{j}}\left(k_{\mathrm{u}}\right) .
$$

The total flow entering a destination link consists of several flows from different origins. The available space in the destination link should be divided over the entering flows, since the total number of vehicles entering the link may not exceed the available space. We divide this available space equally over the different entering flows. When one flow does not fill its part of the space, the remainder is proportionally divided over the rest of the flows. For a detailed description of this process we refer to [54].

\subsection{Interface between the freeway and the urban traffic flow models}

The urban part and the freeway part are coupled via on-ramps and off-ramps. In this section we present the formulas that describe the evolution of the traffic flows on these on-ramps and off-ramps. The main problems are the different simulation time steps $T_{\mathrm{f}}$ and $T_{\mathrm{u}}$ and the boundary conditions that the models create for each other. We assume that the time steps are selected such that $T_{\mathrm{f}} v_{\text {free }, m}<L_{m}$.

\subsubsection{On-ramps}

Consider an on-ramp $r$ that connects intersection $s$ of the urban network to node $p$ of the freeway network, as shown in Figure 3(a). The number of vehicles that enter the on-ramp from the urban network is given by $m_{\text {arr }, s, r}\left(k_{\mathrm{u}}\right)$. These vehicles have a delay $\delta_{s, r}\left(k_{\mathrm{u}}\right)$ similar to (16). The evolution of the queue length is first described with the urban model. At the end of each freeway time step, the queue length as described in the urban model is then translated to the queue length for the freeway model as explained below.

Consider the freeway time step $k_{\mathrm{f}}$ corresponding to the urban time step $k_{\mathrm{u}}=M_{\mathrm{fu}} k_{\mathrm{f}}$ (recall that $T_{\mathrm{f}}=M_{\mathrm{fu}} T_{\mathrm{u}}$ ). In order to get a consistent execution of the urban and freeway models the computations should be done in the following order:

1. Determine the on-ramp departure flow $q_{r, p}\left(k_{\mathrm{f}}\right)$ during the period $\left[k_{\mathrm{f}} T_{\mathrm{f}},\left(k_{\mathrm{f}}+1\right) T_{\mathrm{f}}\right]$ using (8).

2. Assume that these departures spread out evenly over the equivalent urban simulation period $\left[k_{\mathrm{u}} T_{\mathrm{u}},\left(k_{\mathrm{u}}+M_{\mathrm{fu}}\right) T_{\mathrm{u}}\right]$. Compute the departures for each urban time step in this period using $m_{\mathrm{dep}, s, r, p}(k)=\frac{q_{r, p}\left(k_{\mathrm{f}}\right) T_{\mathrm{f}}}{M_{\mathrm{fu}}}$ for $k=k_{\mathrm{u}}, \ldots, k_{\mathrm{u}}+M_{\mathrm{fu}}-1$. 


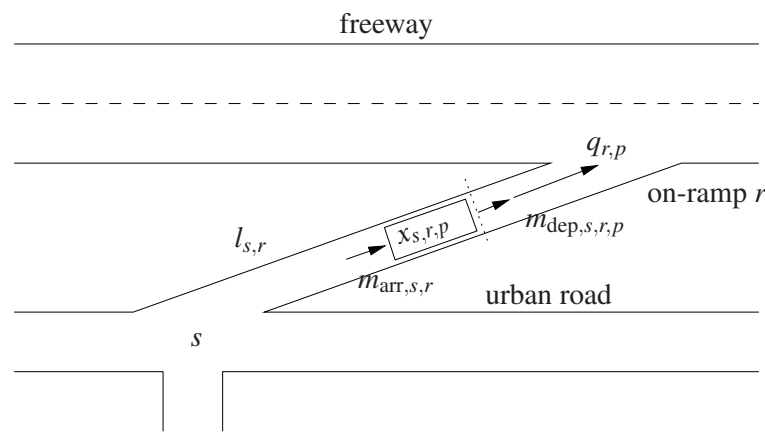

(a) Variables for an on-ramp.

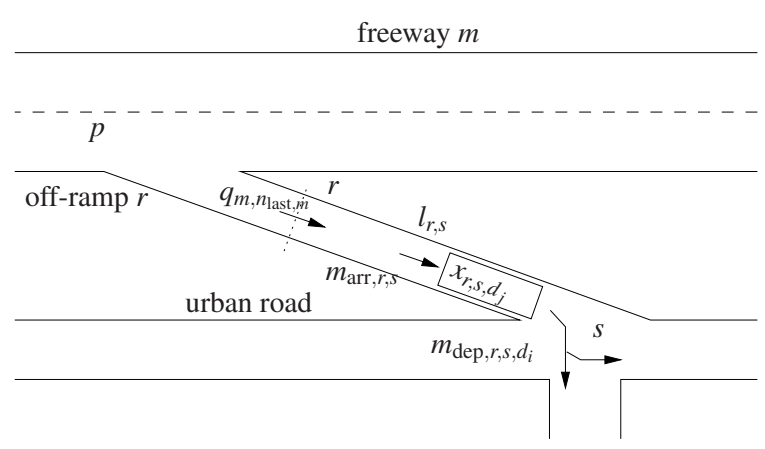

(b) Variables for an off-ramp.

Fig. 3 Overview of variables on on-ramps and off-ramps.

3. The number of arriving vehicles, the free space, and the queue length $x_{s, r, p}$ at link $l_{s, r}$ can now be computed using the equations for the urban traffic model given in Section 2.2.

4. When the queue length $x_{s, r, p}\left(k_{\mathrm{u}}+M_{\mathrm{fu}}\right)$ is computed, we set $w_{o}\left(k_{\mathrm{f}}+1\right)=x_{s, r, p}\left(k_{\mathrm{u}}+\right.$ $\left.M_{\mathrm{fu}}\right)$. It is easy to verify that this is equivalent to (7).

\subsubsection{Off-ramps}

The evolution of the traffic flows on an off-ramp $r$ is computed for the same time steps as for the on-ramp, starting at time step $k_{\mathrm{u}}=M_{\mathrm{fu}} k_{\mathrm{f}}$. The variables are displayed in Figure 3(b). The following steps are required to simulate the evolution of the traffic flows, in order to get a consistent execution of the urban and freeway models:

1. Determine the number of departing vehicles from link $l_{r, s}$ at intersection $s$ during the period $\left[k_{\mathrm{u}} T_{\mathrm{u}},\left(k_{\mathrm{u}}+M_{\mathrm{fu}}\right) T_{\mathrm{u}}\right]$ using the urban traffic flow model.

2. Compute the maximal allowed flow $q_{r, 1}^{\max }\left(k_{\mathrm{f}}\right)$ that can leave the freeway and enter the off-ramp in the period $\left[k_{\mathrm{f}} T_{\mathrm{f}},\left(k_{\mathrm{f}}+1\right) T_{\mathrm{f}}\right]$ based on the available storage space in the link $l_{r, s}$ at the end of the period: 


$$
q_{r, 1}^{\max }\left(k_{\mathrm{f}}\right)=\frac{1}{T_{\mathrm{f}}} S_{r, s}\left(k_{\mathrm{u}}\right)+\sum_{k=k_{\mathrm{u}}}^{k_{\mathrm{u}}+M_{\mathrm{fu}}-1} \sum_{d_{j} \in D_{s}} m_{\mathrm{dep}, r, s, d_{j}}(k) .
$$

The effective outflow $q_{m, n_{\text {last }, m}}\left(k_{\mathrm{f}}\right)$ of freeway link $m$ between node $p$ and offramp $r$ is then given by

$$
q_{m, n_{\text {last }, m}}\left(k_{\mathrm{f}}\right)=\min \left(q_{m, n_{\text {last }, m}^{\text {normal }}}\left(k_{\mathrm{f}}\right), q_{r, 1}^{\max }\left(k_{\mathrm{f}}\right)\right),
$$

where $q_{m, n_{\text {last }, m}}^{\text {normal }}\left(k_{\mathrm{f}}\right)$ is the flow that would have entered the freeway if the off-ramp would not have been blocked.

3. Now the METANET model can be updated for simulation step $k_{\mathrm{f}}+1$.

4. We assume that the outflow of the off-ramp is distributed evenly over the period $\left[k_{\mathrm{f}} T_{\mathrm{f}},\left(k_{\mathrm{f}}+1\right) T_{\mathrm{f}}\right]$ such that

$$
m_{\mathrm{arr}, r, s}\left(k+\delta_{r, s}\right)=\frac{q_{m, n_{\text {last }, m}}\left(k_{\mathrm{f}}\right) T_{\mathrm{f}}}{M_{\mathrm{fu}}} \text { for } k=k_{\mathrm{u}}, \ldots, k_{\mathrm{u}}+M_{\mathrm{fu}}-1 .
$$

The corresponding urban queue lengths $x_{r, s, d_{j}}(k)$ for $k=k_{\mathrm{u}}+1, \ldots, k_{\mathrm{u}}+M_{\mathrm{fu}}$ can be updated using the urban traffic flow model.

\subsection{Emission and fuel consumption model}

In this section we present the dynamic emission and fuel consumption model VTmicro and we show how it can be integrated with the macroscopic METANET traffic flow model of Section 2.1 (see also [59, 60]). A similar approach can also be used to integrate VT-micro with the urban model of Section 2.2.

\subsubsection{Emission and fuel consumption models}

Traffic emission and fuel consumption models calculate the emissions produced and fuel consumed by vehicles based on the operating conditions of the vehicles. The main inputs to the models are the operating conditions of the vehicle (such as speed, acceleration, engine load) [23]. These models can be either average-speed-based or dynamic. Average-speed-based emission and fuel consumption models estimate or predict traffic emission and fuel consumption based on the trip-based average speed of traffic flow [37]. These models can also be used with second-by-second speeds to take some of the variation of the speeds into account [7]. On the contrary, dynamic (or also called microscopic) emission and fuel consumption models use the second-by-second speed and acceleration of individual vehicles to estimate or predict the emissions and the fuel consumption. Such models provide better accuracy than average-speed-based models. Therefore, we will consider a dynamic emission 
and fuel consumption model and integrate it with the macroscopic traffic flow model of Section 2.1.

VT-micro [1] is a microscopic dynamic emission and fuel consumption model that yields emissions and fuel consumption of one individual vehicle using secondby-second speed and acceleration. The model has the form

$$
E_{x}\left(k_{\mathrm{f}}\right)=\exp \left(\tilde{\mathbf{v}}^{\mathrm{T}}\left(k_{\mathrm{f}}\right) \mathbf{P}_{x} \tilde{\mathbf{a}}\left(k_{\mathrm{f}}\right)\right)
$$

where $E_{x}$ is the estimate or prediction of the emission or fuel consumption variable

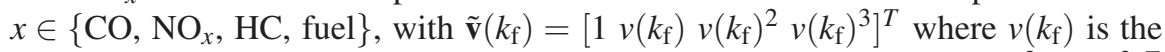

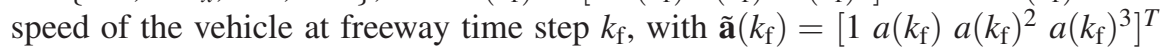
where $a\left(k_{\mathrm{f}}\right)$ is the acceleration of the vehicle at freeway time step $k_{\mathrm{f}}$, and with $\mathbf{P}_{x}$ the model parameter matrix for the variable $x$. The values of the entries of $P_{x}$ for $x \in\left\{\mathrm{CO}, \mathrm{NO}_{x}, \mathrm{HC}\right.$, fuel $\}$ can be found in [1].

The VT-micro emission model does not yield estimates of $\mathrm{CO}_{2}$ emission. But since there is almost an affine relationship between the fuel consumption and the $\mathrm{CO}_{2}$ emission [38], we can compute the $\mathrm{CO}_{2}$ emission as

$$
E_{\mathrm{CO}_{2}}\left(k_{\mathrm{f}}\right)=\delta_{1}+\delta_{2} E_{\text {fuel }}\left(k_{\mathrm{f}}\right),
$$

where $\delta_{1}$ and $\delta_{2}$ are model parameters, the values of which can be found in [38].

Figure 4 depicts the $\mathrm{CO}_{2}$ and fuel consumption versus the vehicle speed for three acceleration values using the equations presented above.

\subsubsection{Integrating METANET with VT-micro}

The VT-micro model is a microscopic traffic emission and fuel consumption model while METANET is a macroscopic traffic flow model. Thus, these two different models are required to be integrated in such a way that VT-micro can get speed and acceleration inputs of the traffic flow from the METANET model at every simulation time step. The speed of the traffic flow can be easily obtained from (4). However, the computation of the acceleration is not as straightforward. In the sequel we show how to obtain the acceleration from the METANET model.

Since the METANET model is discrete in both space and time, there are two acceleration components involved in the model. The first is the "temporal" acceleration of the vehicle flow within a given segment. The second component is the "spatial" acceleration of the vehicles flowing from one segment into another in one simulation time step (see Figure 5).

\section{Temporal acceleration}

The temporal acceleration of vehicles in a segment $i$ of link $m$ at time step $k_{\mathrm{f}}$ is given by: 


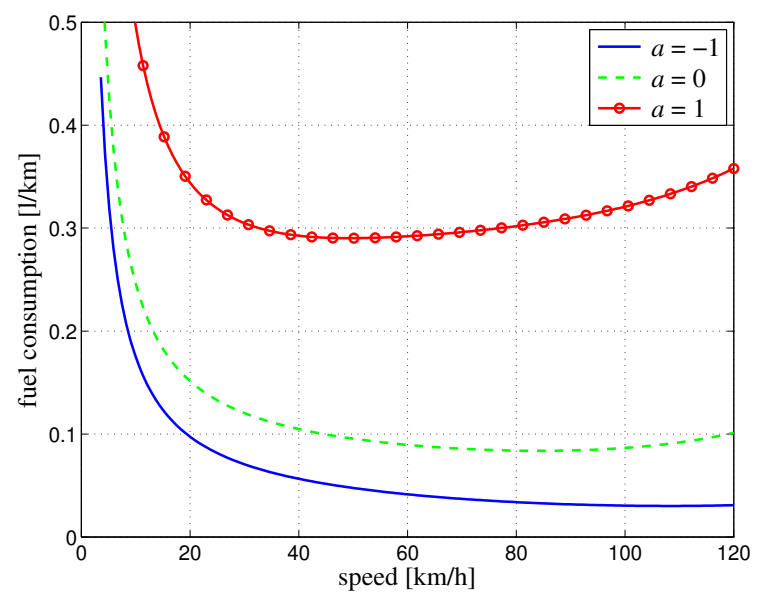

(a) Fuel consumption.

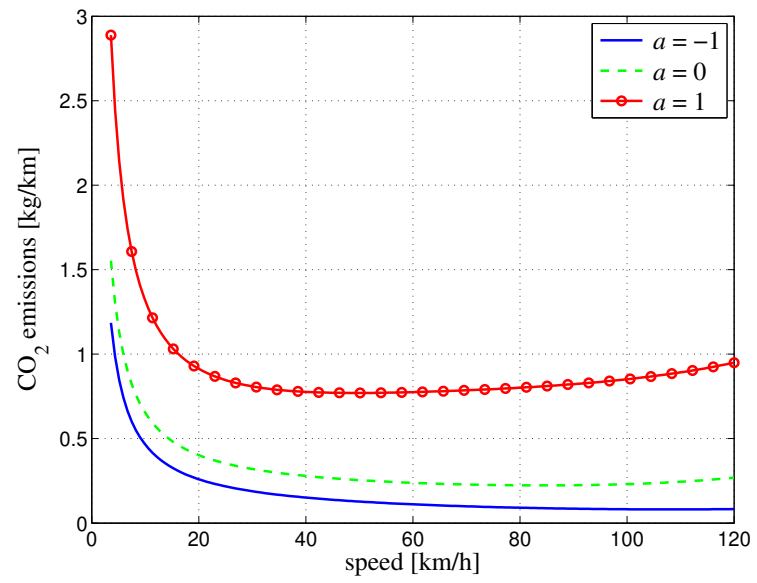

(b) $\mathrm{CO}_{2}$ emissions for diesel fuel.

Fig. 4 Fuel consumption and $\mathrm{CO}_{2}$ emission curves of vehicles as a function of the speed for accelerations $a \in\{-1,0,1\} \mathrm{m} / \mathrm{s}$.

$$
a_{m, i}\left(k_{\mathrm{f}}\right)=\frac{v_{m, i}\left(k_{\mathrm{f}}+1\right)-v_{m, i}\left(k_{\mathrm{f}}\right)}{T_{\mathrm{f}}} .
$$

This equation is the same for all segments of all links.

Let us now determine how many vehicles are subject to this temporal acceleration from time step $k_{\mathrm{f}}$ to time step $k_{\mathrm{f}}+1$. At time step $k_{\mathrm{f}}$ the number of vehicles in segment $i$ of link $m$ is equal to $L_{m} \lambda_{m} \rho_{m, i}\left(k_{\mathrm{f}}\right)$ and from time step $k_{\mathrm{f}}$ to $k_{\mathrm{f}}+1$ (i.e., in the time period $\left.\left[k_{\mathrm{f}} T_{\mathrm{f}},\left(k_{\mathrm{f}}+1\right) T_{\mathrm{f}}\right]\right)$ the number of vehicles leaving segment $i$ is $T_{\mathrm{f}} q_{m, i}\left(k_{\mathrm{f}}\right)$ (see Figure 5). Therefore, the number of vehicles accelerating at the temporal acceleration provided in (25) is 


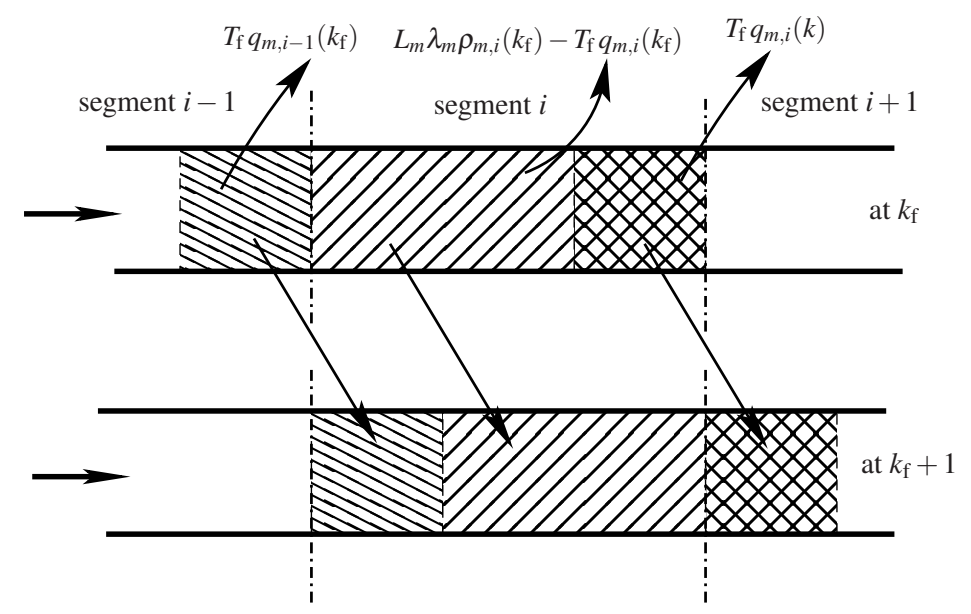

Fig. 5 Illustration of traffic flow in METANET.

$$
n_{m, i}\left(k_{\mathrm{f}}\right)=L_{m} \lambda_{m} \rho_{m, i}\left(k_{\mathrm{f}}\right)-T_{\mathrm{f}} q_{m, i}\left(k_{\mathrm{f}}\right) .
$$

This equation is also the same for all segments of all links.

\section{Spatial acceleration}

The spatial acceleration depends on the geometry of the traffic network. It is different for a link, an on-ramp, an off-ramp, merging links, and splitting links. Here we present the spatial accelerations for a link and an on-ramp. Similar equations can be derived for off-ramps and for splitting and merging links (see [59]).

\section{Link}

In the simulation time step from $k_{\mathrm{f}}$ to $k_{\mathrm{f}}+1$, the spatial acceleration of the vehicles leaving segment $i-1$ of link $m$ and going to segment $i$ of link $m$, and the corresponding number of the vehicles are respectively described by (see also Figure 5):

$$
\begin{aligned}
& a_{m, \Delta i}\left(k_{\mathrm{f}}\right)=\frac{v_{m, i}\left(k_{\mathrm{f}}+1\right)-v_{m, i-1}\left(k_{\mathrm{f}}\right)}{T_{\mathrm{f}}} \\
& n_{m, \Delta i}\left(k_{\mathrm{f}}\right)=T_{\mathrm{f}} q_{m, i-1}\left(k_{\mathrm{f}}\right) .
\end{aligned}
$$




\section{On-ramp}

Consider an on-ramp connected to segment $i$ of link $m$. We assume the initial speed on the on-ramp to be $v_{\text {on }}$. If the inflow of the on-ramp is given by $q_{\text {on, } m, i}\left(k_{\mathrm{f}}\right)$, the spatial acceleration of the vehicles as their speed is changing from $v_{\mathrm{on}}$ to $v_{m, i}\left(k_{\mathrm{f}}+1\right)$ and the corresponding number of vehicles are respectively

$$
\begin{aligned}
& a_{\mathrm{on}, m, \Delta i}\left(k_{\mathrm{f}}\right)=\frac{v_{m, i}\left(k_{\mathrm{f}}+1\right)-v_{\mathrm{on}}}{T_{\mathrm{f}}} \\
& n_{\mathrm{on}, m, \Delta i}\left(k_{\mathrm{f}}\right)=T_{\mathrm{f}} q_{\mathrm{on}, m, i}\left(k_{\mathrm{f}}\right) .
\end{aligned}
$$

\subsubsection{VT-macro}

Recall that as input for the VT-micro model we have to specify speed-acceleration pairs. In all the derivations above both the temporal and spatial accelerations have the form $a_{y}=\frac{v_{2}-v_{1}}{T_{\mathrm{f}}}$. The corresponding speed input is then taken to be $v_{1}$. Furthermore, since the speed-acceleration pair holds for a number of vehicles, the emissions and fuel consumption obtained for the given pair have to be multiplied by the corresponding number of vehicles in order to obtain the total emissions and fuel consumption (as the VT-micro model describes the emissions or fuel consumption of one individual vehicle).

This results in a new traffic emission and fuel consumption model

$$
E_{x, m, i}^{\mathrm{total}}\left(k_{\mathrm{f}}\right)=n^{\mathrm{temp}}\left(k_{\mathrm{f}}\right) E_{x, m, i}^{\mathrm{temp}}\left(k_{\mathrm{f}}\right)+n^{\mathrm{spat}}\left(k_{\mathrm{f}}\right) E_{x, m, \Delta i}^{\mathrm{spat}}\left(k_{\mathrm{f}}\right),
$$

where the superscripts "temp" and "spat" in $n$ and $E$ refer to the temporal or spatial variables, $n$ denotes the number of vehicles, and $E_{x, m, i}$ and $E_{x, m, \Delta i}$ denote the emission or fuel consumption for segment $i$ of link $m$ with respectively the temporal and spatial speed and acceleration inputs. More specifically, $J_{x, m, i}$ and $J_{x, m, \Delta i}$ are computed as in (23) or (24).

We call this new model the VT-macro emission and fuel consumption model.

\section{Model-based predictive traffic control}

In the previous section we have developed a model that describes traffic networks that contain both urban roads and freeways. This model forms the basis for our model-based predictive control method. 


\subsection{Motivation}

To find the optimal combination of traffic control measures (control inputs) we apply a model predictive control (MPC) framework [9, 15, 34]. MPC is an optimal control method applied in a rolling horizon framework. Optimal control has successfully been applied in [29-31] to coordinate or integrate traffic control measures. Both optimal control and MPC have the advantage that the controller generates control signals that are optimal according to a user-supplied objective function. However, MPC has some important advantages over traditional optimal control. First, optimal control has an open-loop structure, which means that the disturbances (in our case: the traffic demands) have to be completely and exactly known in advance, and the traffic model has to be very accurate to ensure sufficient precision for the whole period of operation. MPC operates in closed-loop, which means that the traffic state and the current demands are regularly fed back to the controller, and the controller can take disturbances (here: demand prediction errors) into account and correct for prediction errors resulting from model mismatch. Second, adaptivity is easily implemented in MPC, because the prediction model and/or its parameters can be updated during the operation of the controller. This may be necessary when traffic behavior changes (e.g., in case of incidents, changing weather conditions, lane closures for maintenance). Third, for MPC a shorter prediction horizon is usually sufficient, which reduces complexity, and makes the real-time application of MPC feasible.

\subsection{Principle of operation}

In MPC a discrete-time model is used to predict the future behavior of the traffic network. During a control sampling interval the control signals are taken to be constant. The goal of the controller is to find the control signals that result in an optimal behavior of the traffic flows. To express performance an objective function is defined and the control signals that optimize this function are found via (numerical) optimization.

The control is applied in a rolling-horizon scheme: at each control step $k_{\mathrm{c}}$ (corresponding to the time instant $t=k_{\mathrm{c}} T_{\mathrm{c}}$ with $T_{\mathrm{c}}$ the control time step (typically in the range of 1 to $5 \mathrm{~min}$ for traffic network control)), a new optimization is performed over the prediction horizon $\left[k_{\mathrm{c}} T_{\mathrm{c}},\left(k_{\mathrm{c}}+N_{\mathrm{p}}\right) T_{\mathrm{c}}\right]$, and only the first value of the resulting control signal (the control signal for time instant $k_{\mathrm{c}}$ ) is applied to the process (see Figure 6). At the next control step $k_{\mathrm{c}}+1$ this procedure is repeated. To reduce complexity and improve stability often a control horizon $N_{\mathrm{c}}\left(\leq N_{\mathrm{p}}\right)$ is introduced, and after the control horizon has been passed the control signal is taken to be constant.

So there are two loops in the MPC scheme: the rolling-horizon loop and the optimization loop inside the controller. The loop inside the controller of Figure 6 is executed as many times as required to find the optimal control signals at control time step $k_{\mathrm{c}}$, for given $N_{\mathrm{p}}, N_{\mathrm{c}}$, traffic state, and expected demand. The loop connecting the controller and the traffic system is performed once for each $k_{\mathrm{c}}$ and provides the 


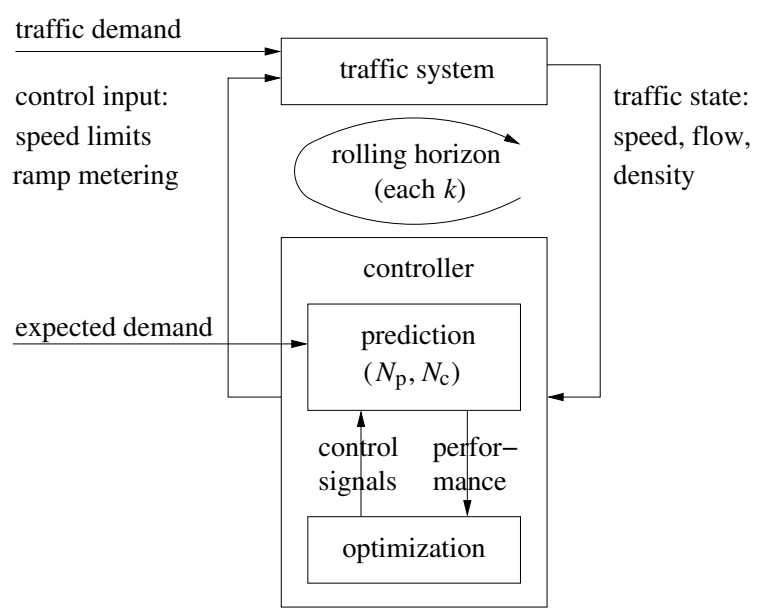

Fig. 6 Schematic view of the model predictive control (MPC) structure.

state feedback to the controller. Recall that this feedback is necessary to correct for (the ever present) prediction errors, and disturbance rejection (compensation for unexpected traffic demand variations). Another advantage of this rolling-horizon approach is that it results in an online adaptive control scheme that allows us to take changes in the system or in the system parameters into account by regularly updating the model of the system.

When optimizing large networks, the computational complexity may become too high. In these cases the network should be separated into some subnetworks that are controlled by separate MPC controllers. The subnetworks should be chosen such that the interaction between them is as small as possible. To handle the remaining interactions (e.g., occasionally overflowing queues) adequately, structures such as hierarchical control or agent-based control can be used (see $[47,55,56])$.

\section{Objective function}

The MPC algorithm has to determine the control signals $\mathbf{c}$ (such as the ramp metering rates, dynamic speed limits, traffic signal settings, etc.) that minimize a given objective function over the period $\left[k_{\mathrm{c}} T_{\mathrm{c}},\left(k_{\mathrm{c}}+N_{\mathrm{p}}\right) T_{\mathrm{c}}\right]$. Possible performance criteria are the total time spent (TTS) in the freeway and the urban part of the network, emission levels, and fuel consumption. Let us now derive the expressions for these performance criteria.

The TTS in the freeway part of the network is given by

$$
J_{\mathrm{TTS}, \mathrm{f}}\left(k_{\mathrm{c}}\right)=T_{\mathrm{f}} \sum_{j \in \mathscr{K}_{\mathrm{f}}\left(k_{\mathrm{c}}, N_{\mathrm{p}}\right)}\left(\sum_{(m, i) \in M_{\mathrm{ls}, \mathrm{f}}} \rho_{m, i}(j) L_{m} \lambda_{m}+\sum_{o \in O_{\mathrm{f}}} w_{o}(j)\right),
$$


where $\mathscr{K}_{\mathrm{f}}\left(k_{\mathrm{c}}, N_{\mathrm{p}}\right)$ is the set of freeway time steps $k_{\mathrm{f}}$ that correspond to the considered prediction period $\left[k_{\mathrm{c}} T_{\mathrm{c}},\left(k_{\mathrm{c}}+N_{\mathrm{p}}\right) T_{\mathrm{c}}\right], M_{\mathrm{ls}, \mathrm{f}}$ is the set of pairs $(m, i)$ of link indices $m$ and segment indices $i$ of the freeway part of the network, and $O_{\mathrm{f}}$ is the set of all mainstream origins and on-ramps at the boundaries of the mixed network (note that on-ramps connecting the urban and the freeway part of the network are considered in the expression for the urban TTS given next).

To compute the TTS for the urban part of the network the number of vehicles in each urban link $n_{\mathrm{veh}, l_{\sigma, s}}$ is required:

$$
n_{\mathrm{veh}, l_{\sigma, s}}\left(k_{\mathrm{u}}\right)=L_{\sigma, s}-S_{\sigma, s}\left(k_{\mathrm{u}}\right),
$$

where $L_{\sigma, s}$ is the maximum number of vehicles that the link can contain. Using this equation the number of vehicles for all urban links, on-ramps, and off-ramps can be determined. The TTS in the urban part of the network is then given by

$$
\begin{array}{r}
J_{\mathrm{TTS}, \mathrm{u}}\left(k_{\mathrm{c}}\right)=T_{\mathrm{u}} \sum_{l \in \mathscr{K}_{\mathrm{u}}\left(k_{\mathrm{c}}, N_{\mathrm{p}}\right)}\left(\sum_{l_{o_{i}, s} \in I_{\mathrm{u}}} n_{\mathrm{veh}, l_{o}, s}(l)+\sum_{l_{s, r} \in R_{\mathrm{on}} \cup R_{\mathrm{off}}} n_{\mathrm{veh}, l_{s, r}}(l)\right. \\
\left.+\sum_{o \in O_{\mathrm{u}}} n_{\mathrm{veh}, o}(l)\right)
\end{array}
$$

where $\mathscr{K}_{\mathrm{u}}\left(k_{\mathrm{c}}, N_{\mathrm{p}}\right)$ is the set of urban time steps $k_{\mathrm{u}}$ that correspond to $\left[k_{\mathrm{c}} T_{\mathrm{c}},\left(k_{\mathrm{c}}+\right.\right.$ $\left.\left.N_{\mathrm{p}}\right) T_{\mathrm{c}}\right], I_{\mathrm{u}}$ is the set of all urban links, $O_{\mathrm{u}}$ is the set of all urban origins $o, R_{\mathrm{on}}$ is the set of urban links $l_{r, s}$ connected to the on-ramps, and $R_{\text {off }}$ is the set of urban links $l_{s, r}$ connected to the off-ramps.

The expression for the emissions and the fuel consumption over the prediction period $\left[k_{\mathrm{c}} T_{\mathrm{c}},\left(k_{\mathrm{c}}+N_{\mathrm{p}}\right) T_{\mathrm{c}}\right]$ is given by

$$
J_{x}\left(k_{\mathrm{c}}\right)=\sum_{j \in \mathscr{K}_{\mathrm{f}}\left(k_{\mathrm{c}}, N_{\mathrm{p}}\right)} \sum_{(m, i) \in M_{\mathrm{ls}, \mathrm{f}}} E_{x, m, i}^{\mathrm{total}}(j)
$$

for $x \in\left\{\mathrm{CO}, \mathrm{NO}_{x}, \mathrm{HC}\right.$, fuel $\}$, where $E_{x, m, i}^{\mathrm{total}}$ is defined by (31).

In order to get smoother control signals, one often also imposes a penalty on the temporal variation of the control signal $\mathbf{c}$ :

$$
J_{\mathrm{var}}^{\mathrm{temp}}\left(k_{\mathrm{c}}\right)=\sum_{j=k_{\mathrm{c}}}^{k_{\mathrm{c}}+N_{\mathrm{c}}-1}\|\mathbf{c}(j)-\mathbf{c}(j-1)\|^{2}
$$

In a similar way, one could also define a penalty on the spatial variation for speed limits $v_{\text {control }, m, i}(j)$ defined on control time steps $j$ :

$$
J_{\text {var }}^{\text {spat }}\left(k_{\mathrm{c}}\right)=\sum_{j=k_{\mathrm{c}}}^{k_{\mathrm{c}}+N_{\mathrm{c}}-1} \sum_{(m, i) \in V_{\mathrm{c}}}\left(v_{\mathrm{control}, m, i}(j)-v_{\mathrm{control}, m, i-1}(j)\right)^{2},
$$


where $V_{\mathrm{c}}$ is the set of pairs of pairs $(m, i)$ of link indices $m$ and segment indices $i$ such that a speed limit is active on both segments $i$ and $i-1$.

The overall objective function used in MPC is then a weighted sum of the above partial objective functions:

$$
J_{\mathrm{MPC}}\left(k_{\mathrm{c}}\right)=\sum_{i=1}^{n} \frac{\gamma_{i}}{J_{\text {nominal }, i}} J_{i}\left(k_{\mathrm{c}}\right),
$$

where $J_{\text {nominal }, i}$ is the nominal value of partial objective function $J_{i}$ (for normalization purposes) and $\gamma_{i}$ is a weighting factor.

\section{Operational constraints}

The constraints may contain upper and lower bounds on the control signal, but also linear or nonlinear equality and inequality constraints on the states of the system. The constraints are used, e.g., to keep the system working within safety limits, or to avoid unwanted situations.

\section{Optimization algorithms}

At each control step the MPC controller computes an optimal control sequence over a given prediction horizon. In general, this optimal control sequence is the solution of a nonlinear, non-convex optimization problem in which the objective function is minimized subject to the model equations and the constraints. To solve this optimization problem different numerical optimization techniques can be applied, such as multi-start sequential quadratic programming (SQP) (see, e.g., [42, Chapter 5]) or pattern search (see, e.g., [44]) for real-valued problems, and genetic algorithms [10], and tabu search [18] or simulated annealing [14] for mixed-integer problems that arise when discrete control measures (e.g., lane closures) are included.

\section{Tuning of $N_{\mathrm{p}}$ and $N_{\mathrm{c}}$}

The tuning rules to select appropriate values for $N_{\mathrm{p}}$ and $N_{\mathrm{c}}$ that have been developed for conventional MPC cannot be applied straightforwardly to the traffic flow control framework presented above. However, based on a heuristic reasoning we can determine an initial guess for these parameters.

The prediction horizon $N_{\mathrm{p}}$ should be larger than the typical travel time from the controlled segments to the exit of the network, because if we take the prediction horizon $N_{\mathrm{p}}$ shorter than the typical travel time in the network, the effect of the vehicles that are influenced by the current control measure and - as a consequence have an effect on the network performance before they exit the network, will not be taken into account. Furthermore, a control action may affect the network state (by 


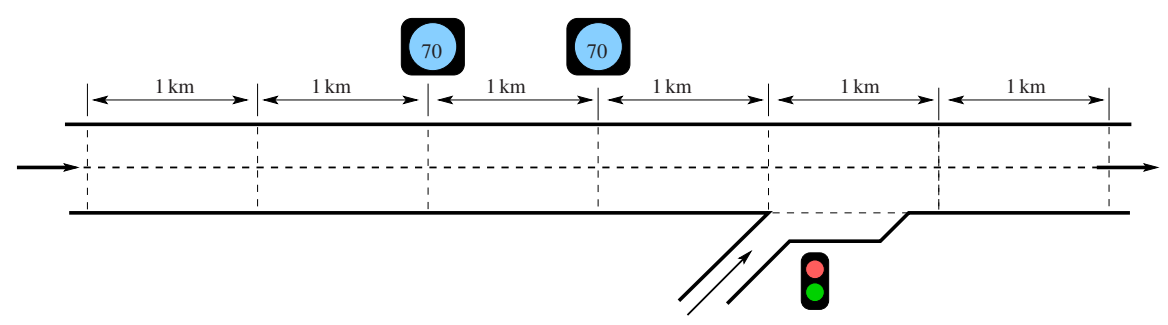

Fig. 7 A $6 \mathrm{~km}$ freeway with metered on-ramp and two dynamic speed limit control.

improved flows, etc.) even when the actually affected vehicles have already exited the network. On the other hand, $N_{\mathrm{p}}$ should not be too large because of the computational complexity of the MPC optimization problem. So based on this reasoning we select $N_{\mathrm{p}}$ to be about the typical travel time in the network.

For the control horizon $N_{\mathrm{c}}$ we select a value that represents a trade-off between the computational effort and the performance.

\section{Case studies}

In order to illustrate the control framework presented above we will now apply it to two case studies: a simple one involving a freeway stretch and a more complex one involving a mixed urban-freeway network.

\subsection{MPC for the reduction of emissions, fuel consumption and travel time on freeways}

\subsubsection{Network and scenario}

Since we want to focus on the relevant points of the approach presented in this chapter, the benchmark network (see Figure 7) for this experiment was chosen as simple as possible. The network consists of one mainstream freeway link with two speed limits, and one metered on-ramp. The on-ramp is located at a distance of $4 \mathrm{~km}$ from the mainstream origin of the freeway link, and it has a capacity of $2000 \mathrm{veh} / \mathrm{h}$. The mainstream freeway link has two lanes with a capacity of $2100 \mathrm{veh} / \mathrm{h}$ each. Segments 3 and 4 of the freeway are equipped with a variable message sign where speed limits can be displayed. The outflow at the end of the freeway is considered to be unrestricted. We assume that the queue length at the on-ramp may not exceed 100 vehicles, in order to prevent spill-back to a surface street intersection.

We use the network parameters as found in [30]: $T_{\mathrm{f}}=10 \mathrm{~s}, \tau=18 \mathrm{~s}, \kappa=$ $40 \mathrm{veh} / \mathrm{km} / \mathrm{lane}, \vartheta=60 \mathrm{~km}^{2} / \mathrm{h}, \rho_{\max }=180 \mathrm{veh} / \mathrm{km} / \mathrm{lane}, a=1.867$, and $\rho_{\text {crit }}=$ 


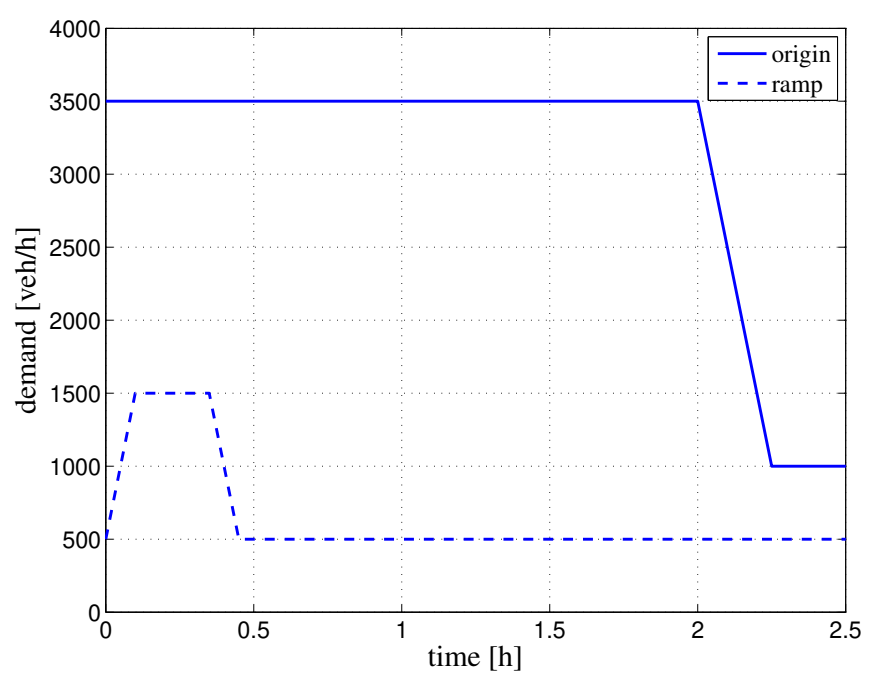

Fig. 8 On-ramp demand (dashed line) and mainstream demand (solid-line) profiles used in the simulations for the first case study.

$33.5 \mathrm{veh} / \mathrm{km} / \mathrm{lane}$. Furthermore, we assume that the desired speed is $10 \%$ higher than the displayed speed limit, that is $\alpha=0.1$.

The controller sampling time $T_{\mathrm{c}}$ is chosen to be $1 \mathrm{~min}$. Moreover, in order to include relevant dynamics of the system states, the prediction horizon and the control horizon are selected to be respectively $N_{\mathrm{p}}=15$ and $N_{\mathrm{c}}=7$.

To examine the effect of the combination of variable speed limits and ramp metering typical demand profiles are considered for the mainstream origin and the onramp (see Figure 8). The mainstream demand has a constant, relatively high level and a drop after $2 \mathrm{~h}$ to a low value in a time span of $15 \mathrm{~min}$. The demand on the on-ramp increases to near capacity, remains constant for $15 \mathrm{~min}$, and decreases finally to a constant low value. For the given demand profiles one uncontrolled (Case 1) and four controlled (Cases 2 to 5) situations are compared. When no control is applied to the system (Case 1), the speed limit is set to a constant value of $102 \mathrm{~km} / \mathrm{h}$ and the ramp rate is constant and equal to 1 . We consider this case as a benchmark to compare the results of the simulations when an MPC controller is implemented. For the controlled cases the objective of the MPC controller is to optimize the following performance criteria (all deemed equally important within each case, i.e., $\gamma_{i}=1$ for all $i$ ):

Case 2: total time spent,

Case 3: total fuel consumption and total time spent,

Case 4: total $\mathrm{NO}_{x}$ emissions and total time spent, and

Case 5: total fuel consumption, total $\mathrm{NO}_{x}$ emissions, and total time spent.

In this experiment the MPC optimization problem is a non-convex, nonlinear problem with real-valued optimization variables (the dynamic speed limits and the 
ramp metering rates). To solve this optimization problem we have selected multistart SQP as optimization algorithm. The SQP algorithm is implemented in the fmincon function of the Matlab optimization toolbox [51].

\subsubsection{Results}

In Table 1 we present the simulation results of the uncontrolled simulation (Case 1) and the controlled simulations (Case 2 to Case 5).

Table 1 Simulation results for the freeway case study.

\begin{tabular}{|c|c|c|c|c|}
\hline \multirow{2}{*}{\multicolumn{2}{|c|}{$\begin{array}{l}\text { Control } \\
\text { objectives }\end{array}$}} & \multicolumn{3}{|c|}{ Simulation results } \\
\hline & & $\begin{array}{c}\text { TTS } \\
(\mathrm{veh} \cdot \mathrm{h})\end{array}$ & $\begin{array}{c}\text { Total } \mathrm{NO}_{x} \\
\text { emissions }(\mathrm{kg})\end{array}$ & $\begin{array}{c}\text { Total fuel } \\
\text { consumption (1) }\end{array}$ \\
\hline Case 1 & : Uncontrolled & 1459 & 8.719 & 6108 \\
\hline Case 2 & : TTS & $1247(-14.6 \%)$ & $8.288(-4.9 \%)$ & $5274(-13.7 \%)$ \\
\hline Case 3 & : TTS + fuel & $1257(-13.9 \%)$ & $8.147(-6.6 \%)$ & $4934(-19.2 \%)$ \\
\hline Case 4 & :TTS $+\mathrm{NO}_{x}$ & $1412(-3.2 \%)$ & $7.654(-12.2 \%)$ & $5290(-13.4 \%)$ \\
\hline Case 5 & $: \mathrm{TTS}+$ fuel $+\mathrm{NO}_{x}$ & $1336(-8.5 \%)$ & $7.786(-10.7 \%)$ & $5088(-16.7 \%)$ \\
\hline
\end{tabular}

When an MPC controller is implemented (Case 2 to Case 5) the values of all the performance indicators are reduced by a certain amount compared to the uncontrolled situation. But, the reduction of the respective performance indicators is dependent on the objective of the controller. As can be seen in the table, when the objective of the controller is to reduce the TTS (Case 2), the TTS is reduced with $14.6 \%$. Moreover, the total $\mathrm{NO}_{x}$ emissions and the total fuel consumption are reduced with $4.9 \%$ and $13.7 \%$ respectively. This indicates that under the given traffic demand and traffic scenario, reducing the TTS can also help in reducing the total $\mathrm{NO}_{x}$ emissions and the total fuel consumption.

When the objective of the controller also includes the total fuel consumption as well as the TTS (Case 3), the results for TTS differ slightly compared to Case 2, while the total fuel consumption shows a significant reduction. So for Case 3 more fuel is saved by making a small sacrifice in the TTS.

In Case 4 the objective of the controller is to reduce the total $\mathrm{NO}_{x}$ emissions and the TTS where both criteria are weighted equally. In this case, the TTS does not show a significant improvement compared to the uncontrolled case. On the other hand, the total $\mathrm{NO}_{x}$ emissions decrease significantly. Moreover, we can see that the total fuel consumption is reduced by $13.4 \%$, which is less than in Case 3. Thus, although the combination of a larger TTS and more fuel consumption indicates inefficient driving behavior in terms of the non-environmental performance indicators (travel time and energy consumption), there is a positive impact on the emissions.

Case 5 encompasses the concerns regarding the travel time, energy consumption, and the environment, as it addresses all three performance indicators by weighting them equally. The simulation results in Table 1 show that in this case the MPC 
controller achieves a balanced trade-off between total time spent, the total fuel consumption, and the total $\mathrm{NO}_{x}$ emissions.

\subsection{MPC for mixed urban-freeway networks}

\subsubsection{Set-up and scenarios}

For this case study a simple network is used, as shown in Figure 9. The network consists of two two-lane freeways (freeway 1 and 2) each with two on-ramps and two off-ramps (ramp 1 to 4). Furthermore, there are two urban intersections (A and $\mathrm{C})$, which are connected to the freeway and to each other. Between these intersections and the freeways there are some crossing roads $(\mathrm{B}, \mathrm{D}$, and $\mathrm{E})$, where there is only crossing traffic that does not turn into other directions, e.g., pedestrian traffic or bicycles. We have selected this network because it contains the most essential elements from mixed networks. There are freeways with on-ramps and off-ramps and controlled intersections not far away from the freeways, resulting in a strong relation between the traffic on the two types of road. The network is small enough to use intuition to analyze and interpret the results, but large enough to make the relevant effects visible.

We will consider four different traffic scenarios all of which are created starting from a "basic" scenario. This basic scenario has a demand of $3600 \mathrm{veh} / \mathrm{h}$ for freeway origins and $1000 \mathrm{veh} / \mathrm{h}$ for urban origins, and turning rates as shown in Figure 9. Each of the scenarios is a variation on this basic scenario, with one variable or parameter changed, or with a constraint added. The total simulated time is $30 \mathrm{~min}$. These are the four scenarios:

Scenario 1: Congestion on the freeway: A traffic jam exists at the downstream end of freeway 1. This congestion grows into the upstream direction and blocks the on-ramps, causing a spill-back leading to urban queues. The congestion is created by imposing a downstream density of $65 \mathrm{veh} / \mathrm{km} /$ lane for the last segment of the freeway.

Scenario 2: Blockage of an urban intersection: On intersection $D$ an incident has occurred, and the whole intersection is blocked. The queues spill back into neighboring intersections, and also block the off-ramps of the freeways. This incident is simulated by setting the saturation flow of all links leaving the intersection to $0 \mathrm{veh} / \mathrm{h}$.

Scenario 3: Rush hour: In this scenario the demand at the origins becomes larger during a short period. We have selected a flow of $500 \mathrm{veh} / \mathrm{h}$ with a peak of $2000 \mathrm{veh} / \mathrm{h}$ for the urban origins, and a flow of $2000 \mathrm{veh} / \mathrm{h}$ with a peak of $4000 \mathrm{veh} / \mathrm{h}$ for freeway origins. The duration of the peak is $10 \mathrm{~min}$.

Scenario 4: Maximum queue length: Here, the queue on the link from intersection A toward intersection B may not become longer than 20 vehicles. 


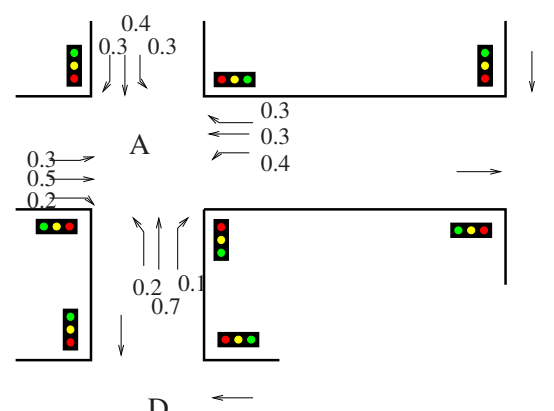

$\mathrm{D} \leftarrow$

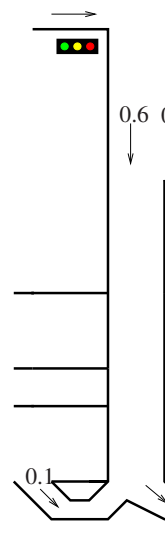

ramp 3

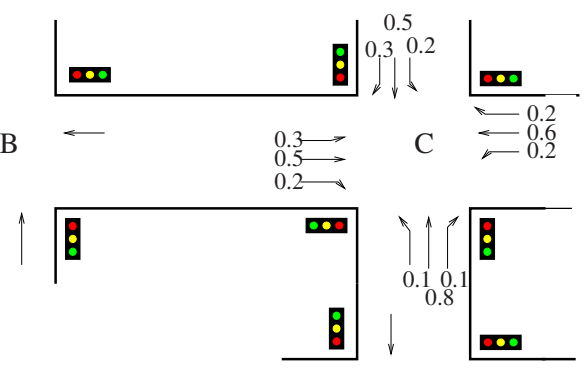

E

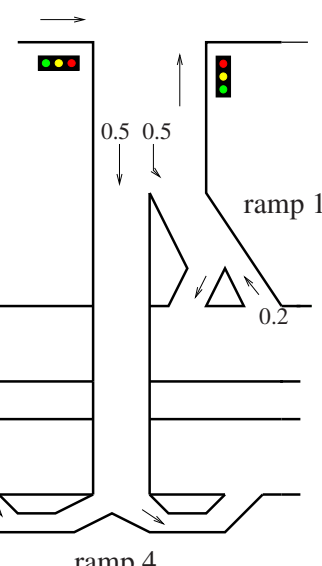

Fig. 9 Network used in the second case study.

This can be a traffic management policy, e.g., when the link is in a residential area.

For all control systems the implementation of the simulations and the controller is completely done in Matlab. We use the traffic flow model described in Section 2 both as the real-world model and as the prediction model. With this set-up we can give a proof of concept of the developed control method, without introducing unnecessary side effects.

In our case study the MPC optimization problem is a non-convex, nonlinear problem with real-valued optimization variables. To solve this optimization problem we have applied multi-start SQP, using the SQP algorithm implemented in the fmincon function of the Matlab optimization toolbox [51].

As cost function we select the total time spent (TTS) in the urban and the freeway part of the network. The model parameters are selected as follows. The parameters of the METANET model are selected according to [30]: $v_{\text {free }, m}=106 \mathrm{~km} / \mathrm{h}, \rho_{\text {crit }, m}=$ $33.5 \mathrm{veh} / \mathrm{km} / \mathrm{lane}, \rho_{\max , m}=180 \mathrm{veh} / \mathrm{km} / \mathrm{lane}, Q_{\text {cap }, m}=4000 \mathrm{veh} / \mathrm{h}, \tau=18 \mathrm{~s}, \kappa=$ $40 \mathrm{veh} / \mathrm{km} / \mathrm{lane}, \vartheta=65 \mathrm{~km}^{2} / \mathrm{h}$, and $a_{m}=1.867$. The parameters of the urban model are: $Q_{\mathrm{cap}, o, s, d}=1000 \mathrm{veh} / \mathrm{h}, L_{\mathrm{av}, \mathrm{veh}}=6 \mathrm{~m}$, and $v_{\mathrm{av}, l_{s, d}}=50 \mathrm{~km} / \mathrm{h}$. 
We have selected the following time steps: $T_{\mathrm{c}}=120 \mathrm{~s}, T_{\mathrm{f}}=10 \mathrm{~s}$, and $T_{\mathrm{u}}=1 \mathrm{~s}$. There are three parameters that can be tuned for the MPC controller. We have selected $N_{\mathrm{p}}=8$ and $N_{\mathrm{c}}=3$ as horizons, and $\gamma_{1}=\gamma_{2}=1$ as weights for the urban and freeway partial performance criteria in the cost function (without normalization in this case).

\subsubsection{Alternative control methods}

Dynamic traffic control systems have already been implemented in the real world. Some examples of these systems are SCATS [58], Toptrac [3], SCOOT [46], UTOPIA/SPOT [43], MOTION [8], and IN-TUC [13].

Here we will use SCOOT and UTOPIA/SPOT to make a comparison between the developed MPC control method and some existing systems. We have selected these methods because they are good representatives of this kind of dynamic traffic control systems. However, these systems are commercial systems, meaning that real specifications are not publicly available. This means that we can only approximate their functioning (see [54] for details). Note that both systems only target the urban traffic. So they optimize the intersections independently of the neighboring freeway.

The main difference between the MPC-based system proposed in this paper and the existing systems like SCOOT and UTOPIA/SPOT is that MPC-based traffic control takes the influences and interactions between the urban and freeway parts of the network into account. By simulating the effect of one measure on both kinds of roads, control settings can be found that provide a trade-off between improving traffic conditions on the freeway and delaying traffic on the urban roads, and vice versa.

Furthermore, the MPC-based system we have developed can handle hard constraints on both the control signals and the states of the traffic network. All control systems can handle constraints that are directly linked to the control signals, e.g., maximal and minimal green times, or maximal cycle times. But the MPC-based system can also handle more indirect constraints such as maximum queue lengths, maximum delays, etc. These constraints are included as hard constraints in the MPC optimization problem, which is subsequently solved using a constrained optimization algorithm. In the other systems such a constraint (like maximum queue lengths) is implemented by adding a penalty term that penalizes the constraint violation to the performance function. This penalty term must become relatively large when the maximum queue length is reached. This results in a very high value of the cost when the maximum queue length is violated. While the purpose of the control is to minimize the cost function, a trade-off will have to be made between minimizing the original cost and violating the queue length constraint. This can lead to either satisfying the constraints with a degraded performance, or violating the constraints and obtaining a better performance. 


\subsubsection{Results}

We have applied SCOOT, UTOPIA/SPOT, and MPC to the case study network for each of the four scenarios. The results are listed in Table 2. This table shows the TTS for the freeway part of the network, for the urban part, and for the whole network. The last column of the table shows the improvement of the MPC method compared to SCOOT (first number) and to UTOPIA/SPOT (second number). This makes it possible to determine in which part of the network the largest improvements are obtained. For the fourth scenario the largest attained queue length is also shown.

The first two scenarios show that the MPC method can improve the performance for the urban as well as for the freeway part of the network when a problem arises in one of the two. The immediate negative effects of such a problem are reduced as well as the negative influence on the rest of the network.

The third scenario shows that the MPC method can control the traffic slightly better that SCOOT and UTOPIA/SPOT when a large peak in the demand occurs. In this scenario the trade-off between the freeway and urban parts of the network can clearly be seen. A reduction of the performance on the urban network can lead to an improvement of the performance on the freeway network, and vice versa. This can be used to obtain a better performance for the total network.

The maximum queue length constraint is implemented in SCOOT and UTOPIA by adding an extra penalty term in the cost function. This term has a relative weight that allows a trade-off between the performance of the network and the importance of the maximum queue length constraints. When the weight is high the queue length constraint is satisfied but the performance is low, as shown in the first simulations done for the fourth scenario. In the second set of simulations the weighting term for the queue constraint is low, resulting in a better performance, but now the maximum queue length is exceeded. The values for MPC are the same for both simulation sets because the queue length constraint is implemented as a hard constraint for the optimization algorithm ${ }^{6}$.

\section{Conclusions}

In this chapter we have shown how model predictive control (MPC) can be used to obtain network-wide coordination and integration of various control measures (e.g., variable speed limits, ramp metering, and traffic signals) as well as a balanced tradeoff between various performance criteria (such as the total time spent, emissions, and fuel consumption). As models are an important component of MPC we have presented an integrated traffic flow model for mixed urban-freeway networks. We have also proposed an emissions and fuel consumption model that can be interfaced

\footnotetext{
6 The MPC-based method violates the constraint with 1 vehicle at the start of the simulation. This is due to infeasibility problems during the optimization, related to the initial state of the network at the start of the simulation. This issue can be solved by increasing the horizons $N_{\mathrm{p}}$ and $N_{\mathrm{c}}$.
} 
Table 2 Results for the second case study: total time spent for the freeway part of the network, for the urban part, and for the total network. The improvement of the MPC-based method compared to SCOOT and UTOPIA/SPOT is also listed.

Scenario 1: Congestion on the freeway.

\begin{tabular}{|l|c|c|c|c|}
\hline & SCOOT & UTOPIA/SPOT & MPC & improvement \\
\hline \hline freeway & 595.4 & 565.1 & 563.9 & $5.3 / 0.3 \%$ \\
urban & 313.6 & 335.7 & 305.7 & $3.0 / 9.0 \%$ \\
\hline total & 909.0 & 900.8 & 869.6 & $4.4 / 3.5 \%$ \\
\hline
\end{tabular}

Scenario 2: Blockage of an urban intersection.

\begin{tabular}{|l|c|c|c|c|}
\hline & SCOOT & UTOPIA/SPOT & MPC & improvement \\
\hline \hline freeway & 498.0 & 526.2 & 495.0 & $0.7 / 6.0 \%$ \\
urban & 665.9 & 672.3 & 620.3 & $6.9 / 7.8 \%$ \\
\hline total & 1163.9 & 1198.5 & 1115.3 & $4.2 / 7.0 \%$ \\
\hline
\end{tabular}

Scenario 3: Rush hour.

\begin{tabular}{|l|c|c|c|c|}
\hline & SCOOT & UTOPIA/SPOT & MPC & improvement \\
\hline \hline freeway & 244.6 & 280.1 & 253.3 & $-3.5 / 9.6 \%$ \\
urban & 409.0 & 383.5 & 386.8 & $5.5 /-1.6 \%$ \\
\hline total & 653.6 & 663.6 & 640.1 & $2.1 / 3.5 \%$ \\
\hline
\end{tabular}

Scenario 4a: Maximum queue length of 20 vehicles, with a large weight.

\begin{tabular}{|l|c|c|c|c|}
\hline & SCOOT & UTOPIA/SPOT & MPC & improvement \\
\hline \hline freeway & 367.2 & 510.3 & 373.9 & $-1.8 / 26.8 \%$ \\
urban & 309.7 & 435.4 & 264.4 & $15.7 / 39.3 \%$ \\
max. queue & 19 & 19 & 21 & \\
\hline total & 676.9 & 945.7 & 638.3 & $6.8 / 32.6 \%$ \\
\hline
\end{tabular}

Scenario 4b: Maximum queue length of 20 vehicles, with a small weight.

\begin{tabular}{|l|c|c|c|c|}
\hline & SCOOT & UTOPIA/SPOT & MPC & improvement \\
\hline freeway & 367.1 & 428.1 & 373.9 & $-1.8 / 13.7 \%$ \\
urban & 303.0 & 360.5 & 264.5 & $13.8 / 26.7 \%$ \\
max. queue & 93 & 43 & 21 & \\
\hline total & 670.1 & 788.6 & 638.3 & $5.8 / 19.1 \%$ \\
\hline
\end{tabular}


with the combined urban-freeway traffic flow model. Finally, we have presented two case studies that illustrate the proposed control approach.

The MPC-based traffic control approach presented in this paper can be extended in various directions. One important topic is the issue of scalability and computational complexity, which could be addressed using a distributed, hierarchical, or multi-agent approach $[47,55,56]$. Other issues include the further development of fast, but accurate traffic flow and emission models for large-scale traffic networks. Moreover, the approach could also be extended to include additional control measures, including those arising in the context of automated highway systems and intelligent vehicle highway systems $[4,5,48,57]$.

The proposed approach is not limited to traffic networks only but could - with proper modifications - also be applied to other types of networks, such as water networks, electricity networks, railway networks, etc. (see also the other chapters of this book).

Acknowledgements Research supported by the BSIK programs "Next Generation Infrastructures (NGI)" and "Towards Sustainable Mobility (TRANSUMO)", the European 7th framework STREP project "Hierarchical and Distributed Model Predictive Control (HD-MPC)" (contract number ICT-INFSO-223854), the European COST Action TU0702, the project "Multi-agent control of large-scale hybrid systems" (DWV.6188) of the Dutch Technology Foundation STW, the NWO-CONNEKT project 014-34-523 "Advanced multi-agent control and information for integrated multi-class traffic networks (AMICI)", the Shell/TU Delft Sustainable Mobility program, the Transport Research Centre Delft, and the Delft Research Center Next Generation Infrastuctures.

We would also like to thank the reviewers for their careful reading of the original manuscript and for their useful feedback and suggestions.

\section{References}

[1] K. Ahn, A.A. Trani, H. Rakha, and M. Van Aerde. Microscopic fuel consumption and emission models. In Proceedings of the 78th Annual Meeting of the Transportation Research Board, Washington, DC, January 1999. CD-ROM.

[2] A. Alessandri, A. Di Febbraro, A. Ferrara, and E. Punta. Nonlinear optimization for freeway control using variable-speed signaling. IEEE Transactions on Vehicular Technology, 48(6):2042-2052, November 1999.

[3] TPA Traffic \& Parking Automation. Toptrac; verkeersafhankelijke regeling voor netwerken. Technical report, TPA Traffic \& Parking Automation, NHTV Breda, The Netherlands, 2002. In Dutch.

[4] L.D. Baskar, B. De Schutter, and H. Hellendoorn. Hierarchical traffic control and management with intelligent vehicles. In Proceedings of the 2007 IEEE Intelligent Vehicles Symposium (IV'07), pages 834-839, Istanbul, Turkey, June 2007.

[5] L.D. Baskar, B. De Schutter, and H. Hellendoorn. Model predictive control for intelligent speed adaptation in intelligent vehicle highway systems. In Pro- 
ceedings of the 17th IEEE International Conference on Control Applications, pages 468-473, San Antonio, Texas, September 2008.

[6] T. Bellemans. Traffic Control on Motorways. PhD thesis, Faculty of Applied Sciences, K.U.Leuven, Leuven, Belgium, May 2003.

[7] P.G. Boulter, T. Barlow, I.S. McCrae, S. Latham, D. Elst, and E. van der Burgwal. Road traffic characteristics, driving patterns and emission factors for congested situations. Technical report, TNO Automotive, Department Powertrains-Environmental Studies \& Testing, Delft, The Netherlands, 2002. OSCAR Deliverable 5.2.

[8] F. Busch and G. Kruse. MOTION for SITRAFFIC - A modern approach to urban traffic control. In Proceedings of the IEEE Conference on Intelligent Transportation Systems, pages 61-64, Oakland, California, 2001.

[9] E.F. Camacho and C. Bordons. Model Predictive Control in the Process Industry. Springer-Verlag, Berlin, Germany, 1995.

[10] L. Davis, editor. Handbook of Genetic Algorithms. Van Nostrand Reinhold, New York, New York, 1991.

[11] F.P. Deflorio. Evaluation of a reactive dynamic route guidance strategy. Transportation Research Part C, 11(5):375-388, October 2003.

[12] C. Diakaki, V. Dinopoulou, K. Aboudolas, and M. Papageorgiou. Signal management in real time for urban traffic networks. Technical report, Technical University of Crete - IST programme, Chania, Greece, July 2002.

[13] C. Diakaki, M. Papageorgiou, and T. McLean. Integrated traffic-responsive urban corridor control strategy in Glasgow, Scotland: Application and evaluation. Transportation Research Record, (1727):101-111, 2000.

[14] R.W. Eglese. Simulated annealing: A tool for operations research. European Journal of Operational Research, 46(3):271-281, June 1990.

[15] C.E. García, D.M. Prett, and M. Morari. Model predictive control: Theory and practice - A survey. Automatica, 25(3):335-348, May 1989.

[16] N.H. Gartner. Development of demand-responsive strategies for urban traffic control. In P. Thoft-Christensen, editor, Proceedings of the 11th IFIP Conference on System Modelling and Optimization, pages 166-174. New York, New York: Springer-Verlag, 1984.

[17] N.H. Gartner, J.D.C. Little, and H. Gabbay. Simultaneous optimization of offsets, splits, and cycle time. Transportation Research Record, (596):6-15, 1976.

[18] F. Glover and M. Laguna. Tabu Search. Kluwer Academic Publishers, Boston, Massachusetts, 1997.

[19] A. Hegyi. Model Predictive Control for Integrating Traffic Control Measures. $\mathrm{PhD}$ thesis, Delft University of Technology, Delft, The Netherlands, February 2004. TRAIL Thesis Series T2004/2.

[20] A. Hegyi, B. De Schutter, and H. Hellendoorn. Model predictive control for optimal coordination of ramp metering and variable speed limits. Transportation Research Part C, 13(3):185-209, June 2005. 
[21] A. Hegyi, B. De Schutter, and J. Hellendoorn. Optimal coordination of variable speed limits to suppress shock waves. IEEE Transactions on Intelligent Transportation Systems, 6(1):102-112, March 2005.

[22] D. Helbing, A. Hennecke, V. Shvetsov, and M. Treiber. Micro- and macrosimulation of freeway traffic. Mathematical and Computer Modelling, 35(56):517-547, March 2002.

[23] J. Heywood. Internal Combustion Engine Fundamentals. McGraw-Hill, New York, New York, 1988.

[24] S.P. Hoogendoorn and P.H.L. Bovy. Generic gas-kinetic traffic systems modeling with applications to vehicular traffic flow. Transportation Research Part B, 35(4):317-336, May 2001.

[25] S.P. Hoogendoorn and P.H.L. Bovy. State-of-the-art of vehicular traffic flow modelling. Proceedings of the Institution of Mechanical Engineers, Part I: Journal of Systems and Control Engineering, 215(4):283-303, August 2001.

[26] A. Karimi, A. Hegyi, B. De Schutter, J. Hellendoorn, and F. Middelham. Integrated model predictive control of dynamic route guidance information systems and ramp metering. In Proceedings of the 7th International IEEE Conference on Intelligent Transportation Systems (ITSC 2004), pages 491-496, Washington, DC, October 2004.

[27] H.R. Kashani and G.N. Saridis. Intelligent control for urban traffic systems. Automatica, 19(2):191-197, March 1983.

[28] A. Kotsialos and M. Papageorgiou. Motorway network traffic control systems. European Journal of Operational Research, 152(2):321-333, January 2004.

[29] A. Kotsialos, M. Papageorgiou, M. Mangeas, and H. Haj-Salem. Coordinated and integrated control of motorway networks via non-linear optimal control. Transportation Research Part C, 10(1):65-84, February 2002.

[30] A. Kotsialos, M. Papageorgiou, and A. Messmer. Optimal coordinated and integrated motorway network traffic control. In Proceedings of the 14th International Symposium of Transportation and Traffic Theory (ISTTT), pages 621-644, Jerusalem, Israel, July 1999.

[31] A. Kotsialos, M. Papageorgiou, and F. Middelham. Optimal coordinated ramp metering with advanced motorway optimal control. In Proceedings of the 80th Annual Meeting of the Transportation Research Board, Washington, DC, 2001. Paper no. 01-3125.

[32] H. Lenz, R. Sollacher, and M. Lang. Nonlinear speed-control for a continuum theory of traffic flow. In Proceedings of the 14th IFAC World Congress (IFAC'99), volume Q, pages 67-72, Beijing, China, January 1999.

[33] M.J. Lighthill and G.B. Whitham. On kinematic waves: I. Flood movement in long rivers. Proceedings of the Royal Society of London, 299A:281-316, May 1955.

[34] J.M. Maciejowski. Predictive Control with Constraints. Prentice Hall, Harlow, UK, 2002.

[35] A. Messmer and M. Papageorgiou. METANET: A macroscopic simulation program for motorway networks. Traffic Engineering and Control, 31(8/9):466-470, August/September 1990. 
[36] Ministry of Transport, Public Works and Watermanagement. Mobiliteitsonderzoek Nederland 2005, tabellenboek. Technical report, AVV transport research center, Rotterdam, The Netherlands, 2006. In Dutch.

[37] L. Ntziachristos and Z. Samaras. Speed-dependent representative emission factors for catalyst passanger cars and influencing parameters. Atmospheric Environment, 34(27):4611-4619, March 2000.

[38] M.T. Oliver-Hoyo and G. Pinto. Using the relationship between vehicle fuel consumption and $\mathrm{CO}_{2}$ emissions to illustrate chemical principles. Journal of Chemical Education, 85(2):218-220, February 2008.

[39] M. Papageorgiou, J.M. Blosseville, and H. Haj-Salem. Modelling and realtime control of traffic flow on the southern part of Boulevard Périphérique in Paris: Part II: Coordinated on-ramp metering. Transportation Research Part A, 24(5):361-370, September 1990.

[40] M. Papageorgiou, H. Hadj-Salem, and J.M. Blosseville. ALINEA: A local feedback control law for on-ramp metering. Transportation Research Record, (1320):58-64, 1991.

[41] M. Papageorgiou and A. Kotsialos. Freeway ramp metering: An overview. IEEE Transactions on Intelligent Transportation Systems, 3(4):271-280, December 2002.

[42] P.M. Pardalos and M.G.C. Resende, editors. Handbook of Applied Optimization. Oxford University Press, Oxford, UK, 2002.

[43] Peek Traffic. UTOPIA/SPOT - Technical Reference Manual. Peek Traffic Scandinavia, January 2002.

[44] D.A. Pierre. Optimization Theory with Applications. Dover Publications, New York, New York, 1986.

[45] P.I. Richards. Shock waves on the highway. Operations Research, 4(1):42-57, January-February 1956.

[46] D.I. Robertson and R.D. Bretherton. Optimizing networks of traffic signals in real time - the SCOOT method. IEEE Transactions on Vehicular Technology, 40(1):11-15, February 1991.

[47] R. Schleiffer, ed. Special Issue on Intelligent Agents in Traffic and Transportation. Transportation Research Part C, 10(5-6):325-527, October-December 2002.

[48] S.E. Shladover, C.A. Desoer, J.K. Hedrick, M. Tomizuka, J. Walrand, W.B. Zhang, D.H. McMahon, H. Peng, S. Sheikholesham, and N. McKeown. Automatic vehicle control developments in the PATH program. IEEE Transactions on Vehicular Technology, 40(1):114-130, February 1991.

[49] S. Smulders. Control of freeway traffic flow by variable speed signs. Transportation Research Part B, 24(2):111-132, April 1990.

[50] H. Taale and F. Middelham. Ten years of ramp-metering in The Netherlands. In Proceedings of the 10th International Conference on Road Transport Information and Control, pages 106-110, London, UK, April 2000.

[51] The MathWorks, Natick, Massachusetts. Optimization Toolbox 4 - User's Guide, March 2009. Revised for Version 4.2. 
[52] M. van den Berg, B. De Schutter, A. Hegyi, and J. Hellendoorn. Model predictive control for mixed urban and freeway networks. In Proceedings of the 83rd Annual Meeting of the Transportation Research Board, Washington, DC, January 2004. Paper 04-3327.

[53] M. van den Berg, A. Hegyi, B. De Schutter, and J. Hellendoorn. A macroscopic traffic flow model for integrated control of freeway and urban traffic networks. In Proceedings of the 42nd IEEE Conference on Decision and Control, pages 2774-2779, Maui, Hawaii, December 2003.

[54] M. van den Berg, A. Hegyi, B. De Schutter, and J. Hellendoorn. Integrated traffic control for mixed urban and freeway networks: A model predictive control approach. European Journal of Transport and Infrastructure Research, 7(3):223-250, September 2007.

[55] R.T. van Katwijk. Multi-Agent Look-Ahead Traffic Adaptive Control. PhD thesis, Delft University of Technology, Delft, The Netherlands, January 2008. TRAIL Thesis Series T2008/3.

[56] R.T. van Katwijk, P. van Koningsbruggen, B. De Schutter, and J. Hellendoorn. Test bed for multiagent control systems in road traffic management. Transportation Research Record, (1910):108-115, 2005.

[57] P. Varaiya. Smart cars on smart roads: Problems of control. IEEE Transactions on Automatic Control, 38(2):195-207, February 1993.

[58] B. Wolshon and W.C. Taylor. Analysis of intersection delay under real-time adaptive signal control. Transportation Research Part C, 7(1):53-72, February 1999.

[59] S.K. Zegeye, B. De Schutter, H. Hellendoorn, and E. Breunesse. Integrated macroscopic traffic flow and emission model based on METANET and VTmicro. In Proceedings of the International Conference on Models and Technologies for Intelligent Transportation Systems, Rome, Italy, June 2009.

[60] S.K. Zegeye, B. De Schutter, H. Hellendoorn, and E. Breunesse. Reduction of travel times and traffic emissions using model predictive control. In Proceedings of the 2009 American Control Conference, St. Louis, Missouri, June 2009. 